\title{
A conserved neuropeptide system links head and body motor circuits to enable adaptive behavior
}

Shankar Ramachandran ${ }^{1, \#}$, Navonil Banerjee ${ }^{1, \#, a}$, Raja Bhattacharya ${ }^{1, \#, b}$, Michele L Lemons ${ }^{2}$, Jeremy Florman ${ }^{1}$, Christopher M. Lambert ${ }^{1}$, Denis Touroutine ${ }^{1}$, Kellianne Alexander ${ }^{1}$, Liliane Schoofs ${ }^{3}$, Mark J Alkema $^{1}$, Isabel Beets ${ }^{3}$ and Michael M. Francis ${ }^{1 *}$

${ }^{1}$ Department of Neurobiology

715 Lazare Research Building

University of Massachusetts Medical School

364 Plantation St.

Worcester, MA 01605

${ }^{2}$ Department of Biological and Physical Sciences

Assumption University

Worcester, MA 01609

${ }^{3}$ Department of Biology

University of Leuven (KU Leuven)

Leuven, Belgium

*corresponding author (michael.francis@umassmed.edu)

\#equal contributions

Current address:

aDepartment of Microbiology, Immunology, and Molecular Genetics, University of California, Los Angeles, Los Angeles, CA

${ }^{\mathrm{b}}$ Amity Institute of Biotechnology, Amity University Kolkata, West Bengal, India 


\section{SUMMARY}

Neuromodulators promote adaptive behaviors that are often complex and involve concerted activity changes across circuits that are not physically connected. It is not well understood how neuromodulatory systems act across circuits to elicit complex behavioral responses. Here we show that the C. elegans NLP-12 neuropeptide system, related to the mammalian cholecystokinin system, shapes responses to food availability by modulating the activity of head and body wall motor neurons. NLP-12 modulation is achieved through conditional involvement of alternate GPCR targets. The CKR-1 GPCR is highly expressed in the head motor circuit, and enhances head bending and trajectory changes during local food searching, primarily through stimulatory actions on SMD head motor neurons. Under basal conditions, NLP-12 signaling regulates body bending, primarily through the CKR-2 GPCR located on body wall motor neurons. Thus, locomotor responses to changing environmental conditions emerge from conditional NLP-12 stimulation of head or body wall motor neurons.

Impact statement: Investigation of neuromodulatory control of ethologically conserved arearestricted food search behavior shows that NLP-12 stimulation of the head motor circuit through the previously uncharacterized CKR-1 GPCR promotes food searching.

\section{Key words:}

Neuropeptide, neuromodulation, neural circuits, adaptive behavior, area-restricted food search, C. elegans, G protein-coupled receptor 


\section{Author contributions}

$\mathrm{RB}$ and MMF were responsible for original conceptualization and design of the project, NB and SR further developed the project. SR, NB and RB carried out plasmid and strain building, experimentation and analysis. DT built plasmids and strains. CML generated molecular biology constructs. IB and LS performed in vitro experiments and analysis. ML, KA, JF and MJA assisted with behavior and calcium imaging experiments. MMF was responsible for data interpretation and writing the manuscript with SR.

\section{Acknowledgements}

We thank the Caenorhabditis Genetics Center, which is funded by the National Institutes of Health National Center for Research Resources, and the Mitani laboratory (National Bioresource Project) for providing Caenorhabditis elegans strains. We thank Mei Zhen lab for Matlab script for calcium imaging analysis, Claire Bénard for strains, Michael Gorczyca and William Joyce for technical support. We thank Francis lab members for helpful comments on the manuscript.

\section{Funding}

This work was supported by NIH R21NS093492 (MMF), European Research Council 340318 and Research Foundation Flanders grant G0C0618N (IB).

Disclosure Statement: The authors have nothing to declare. 


\section{Introduction}

Neuromodulators serve critical roles in altering the functions of neurons to elicit alternate behavior. Disruptions in neuromodulatory transmitter systems are associated with a variety of behavioral and neuropsychiatric conditions, including eating disorders, anxiety, stress and mood disorders, depression, and schizophrenia. ${ }^{1-3}$ To achieve their effects, neuromodulatory systems may act broadly through projections across many brain regions or have circuit-specific actions, based on the GPCRs involved and their cellular expression. A single neuromodulator may therefore perform vastly different signaling functions across the circuits where it is released. For example, Neuropeptide Y (NPY) coordinates a variety of energy and feeding-related behaviors in mammals through circuit-specific mechanisms. NPY signaling may increase or decrease food intake depending upon the circuit and GPCR targets involved. 4,5 Due to the varied actions of neuromodulators across cell types and neural circuits, it has remained challenging to define how specific neuromodulatory systems act in vivo to elicit alternate behaviors. Addressing this question in the mammalian brain is further complicated by the often widespread and complex projection patterns of neuromodulatory transmitter systems, and our still growing knowledge of brain connectivity.

The compact neural organization and robust genetics of invertebrate systems such as Caenorhabditis elegans are attractive features for studies of neuromodulatory function. Prior work has shown that $C$. elegans NLP-12 neuropeptides are key modulatory signals in the control of behavioral adaptations to changing environmental conditions, such as food availability or oxygen abundance. ${ }^{6-8}$ The NLP-12 system is the closest relative of the mammalian Cholecystokinin (CCK) neuropeptide system and is highly conserved across flies, worms and mammals. ${ }^{9-11}$ CCK is abundantly expressed in the mammalian brain, however a clear understanding of the regulatory actions of CCK on the circuits where it is expressed is only now beginning to emerge. ${ }^{12-15}$ Like mammals, the $C$. elegans genome encodes two putative CCK-responsive G protein-coupled receptors (GPCRs) (CKR-1 and CKR-2), 
though, to date, direct activation by NLP-12 peptides has only been demonstrated for the CKR-2 GPCR. ${ }^{9-11,16}$ The experimental tractability of C. elegans, combined with the highly conserved nature of the NLP-12/CCK system, offers a complementary approach for uncovering circuit-level actions underlying neuropeptide modulation, in particular NLP-12/CCK neuropeptide signaling.

Sudden decreases in food availability or environmental oxygen levels each evoke a characteristic behavioral response in $C$. elegans where animals limit their movement to a restricted area by increasing the frequency of trajectory changes (reorientations), a behavior known as local or arearestricted searching (ARS). ARS is a highly conserved adaptive behavior and is evident across diverse animal species. ${ }^{17-23}$ ARS responses during food searching in particular are rapid and transient. Trajectory changes increase within a few minutes after food removal, and decrease with prolonged removal from food (>15-20 minutes) as animals transition to global searching (dispersal). ${ }^{6-8,24-27}$ The clearly discernible behavioral states during food searching present a highly tractable model for understanding contributions of specific neuromodulatory systems. NLP-12 neuropeptide signaling promotes increases in body bending amplitude and turning during movement, ${ }^{6,7}$ motor adaptations that are particularly relevant for ARS. Notably, $n / p-12$ is strongly expressed in only a single neuron, the interneuron DVA that has synaptic targets in the motor circuit and elsewhere. 6,28 Despite the restricted expression of $n / p-12$, there remains considerable uncertainty about the cellular targets of NLP-12 peptides and the circuit-level mechanisms by which NLP-12 modulation promotes its behavioral effects.

Here we explore the GPCR and cellular targets involved in NLP-12 neuromodulation of local food searching. Our findings reveal a primary requirement for NLP-12 signaling onto SMD head motor neurons, mediated through the CKR-1 GPCR, for trajectory changes during local searching. In contrast, NLP-12 signaling through both CKR-1 and CKR-2 GPCRs contribute to NLP-12 regulation of 
basal locomotion, likely through signaling onto head and body wall motor neurons. Our results suggest a model where NLP-12 signaling acts through CKR-1 and CKR-2 to coordinate activity changes across head and body wall motor circuits during transitions between basal and adaptive motor states.

\section{Results}

\section{NLP-12/CCK induced locomotor responses require functional CKR-1 signaling}

To decipher mechanisms underlying NLP-12 regulation of local food searching, we sought to identify genes required for NLP-12-mediated locomotor changes, in particular the G protein-coupled receptors (GPCRs) responsible for NLP-12 signaling. The C. elegans genome encodes closely related CKR-1 and CKR-2 (holecystokinin-like Receptors 1 and 2) GPCRs with sequence homology to the mammalian Cholecystokinin receptors CCK-1 and CCK-2 (Fig. S1A-B). ${ }^{9-11}$ Prior work demonstrated that NLP-12 activates CKR-2 in vitro. ${ }^{9}$ Further genetic studies provided evidence that NLP-12 signaling mediates functional plasticity at cholinergic neuromuscular synapses through CKR-2 modulation of acetylcholine release from motor neurons. $6,29,30$ Surprisingly however, deletion of $c k r-2$ does not strongly affect local search behavior. ${ }^{6}$ As functional roles for the CKR-1 GPCR have not been previously described, we sought to determine whether CKR-1 may be acting either alone or in combination with CKR-2 to direct NLP-12 regulation of local searching. We first isolated a full-length $c k r-1$ cDNA identical to the predicted $c k r-1$ sequence. As expected, we found the $c k r-1$ locus encodes a predicted protein containing 7 transmembrane domains and sharing strong similarity to the CCK-like GPCR family (Fig. S1).

To define potential roles for CKR-1 and CKR-2 in local searching, we took advantage of a strain we had previously generated that stably expresses high levels of the NLP-12 precursor [nlp-12(OE)]. ${ }^{6}$ Overexpression of $n / p-12$ in this manner elicits exaggerated loopy movement, increased trajectory changes and enhanced body bend amplitude (Fig. 1A, 6C, Video S1). The average amplitude of 
bending is increased approximately 3 -fold in comparison to wild type (Fig. 1B), and body bends are more broadly distributed over steeper angles (Fig. 1C-D). These overexpression effects are constitutive, offering experimental advantages for pursuing genetic strategies to identify signaling mechanisms. We investigated the requirement for CKR-1 and CKR-2 in the locomotor changes elicited by $n / p-12$ overexpression using available strains carrying independent deletions in each of these genes. The $c k r-2$ deletion ( $t m 3082)$ has been characterized previously and likely represents a null allele.9,11,29 The $c k r-1$ deletion (ok2502) removes 1289 base pairs, including exons 3-7 that encode predicted transmembrane domains 2-5 (Fig. S1B-C) and therefore also likely represents a null allele. $c k r-1$ and $c k r-2$ single gene deletions each partially reversed the effects of $n l p-12$ overexpression (Fig. $1 A, B, D, 6 C)$, indicating that both CKR-1 and CKR-2 GPCRs are active under conditions when NLP-12 peptides are present at high levels. Notably, $c k r-1$ deletion showed slightly greater suppression of $n / p-$ 12(OE) phenotypes compared with $c k r-2$ deletion (Fig. 1B,D, 6C). Combined deletion of $c k r-1$ and $c k r-$ 2 largely reversed the locomotor changes produced by NLP-12 overexpression (Fig. 1A,B,D, 6C), indicating that the GPCRs act in a partially redundant manner. Our genetic analysis of nlp-12 overexpression confirms a role for the CKR-2 GPCR in NLP-12-elicited motor adaptations, and importantly, provides first evidence implicating the previously uncharacterized CKR-1 GPCR in NLP-12 modulation of motor activity.

\section{NLP-12 activates CKR-1 with high potency}

To obtain direct evidence for NLP-12 activation of CKR-1, we used an in vitro bioluminescence-based approach. CKR-1 was expressed in Chinese hamster ovarian (CHO) cells stably expressing the promiscuous G-protein alpha subunit $\mathrm{G}_{\alpha 16}$ and a bioluminescent calcium indicator, aequorin. ${ }^{31}$ The NLP-12 precursor gives rise to 2 distinct mature peptides, NLP-12-1 and NLP-12-2. Application of either NLP-12-1 or NLP-12-2 synthetic peptides produced robust calcium responses in cells expressing CKR-1. These responses were concentration-dependent with $\mathrm{EC}_{50}$ values of 3.5 and 1.9 
nM for NLP-12-1 and NLP-12-2 peptides, respectively (Fig. 1E). These EC $_{50}$ values are comparable to those measured for NLP-12 activation of CKR-2 (8.0 nM and 10.2 nM) (Fig. 1F) ${ }^{9}$, suggesting NLP-12 peptides act with similar potency across CKR-1 and CKR-2 GPCRs. Importantly, no other peptides from a library of over 350 synthetic C. elegans peptides elicited CKR-1 activation, nor did the NLP-12 peptides evoke calcium responses in cells transfected with empty vector (Fig. S2), indicating that CKR-1, like CKR-2, is a highly specific receptor for NLP-12.

\section{CKR-1 is a key signaling component for local search behavior}

To more deeply investigate roles for CKR-1 and CKR-2 in NLP-12 regulation of movement, we quantified body and head bending during basal locomotion (in the presence of food) using single worm tracking analysis. $n / p-12$ deletion significantly reduced both body bending and head bending angles in comparison to wild type (Fig. 2A-B). Similarly, single deletions in $c k r-1$ and $c k r-2$ each produced significant reductions in body bending, and combined deletion produced effects similar to nlp-12 deletion (Fig. 2A). In contrast, head bending was strikingly affected by $c k r-1$ deletion, while $c k r-2$ deletion did not produce a significant reduction (Fig. 2B). The preferential involvement of CKR-1 in head bending suggested the interesting possibility that CKR-1 and CKR-2 GPCRs differentially regulate specific features of locomotion.

To explore this possibility further, we investigated the involvement of CKR-1 and CKR-2 GPCRs in local search responses following removal from food. Specifically, we monitored worm movement during a 35-minute period immediately after removal from food and quantified turning behavior during the first (0-5, local searching, Video S2) and last (30-35, dispersal, Video S3) five minutes (Fig. 3A). Post-hoc video analysis proved most reliable for measuring turning behavior during local searching.

We quantified changes in trajectory (reorientations), that resulted in a change of $>50^{\circ}$ in the direction of movement, executed either through forward turns or reversal-coupled omega turns [Fig. 3B, S3]. For wild type, we noted an increase in reorientations immediately following removal from food 
compared to animals maintained on food (Fig. S4A). Consistent with our previous findings ${ }^{6}$, we found that deletion of $n l p-12$ significantly decreased reorientations immediately following removal from food (Fig. 3C-D). In particular, we noted a significant reduction in the forward reorientations of nlp-12 mutants, but no appreciable effect on reversal-coupled omega turns (Fig. S4B). Deletion of ckr-2 produced no appreciable effect on reorientations (Fig. 3C-D, ${ }^{6}$ ); however, single deletion of $c k r-1$ decreased reorientations to a similar level as observed for $n / p-12$ deletion (Fig. 3C-D). Similar to $n / p$ 12(If), we found that $c k r-1(I f)$ significantly impacted forward reorientations, but did not affect reversalcoupled omega turns (Fig. S4B). Combined deletion of $c k r-1$ and $c k r-2$ provided no additional decrease beyond that observed for single $c k r-1$ deletion (Fig. 3C-D). In addition, combined deletion of nlp-12 and $c k r-1$ did not further decrease reorientations compared with either of the single mutants (Fig. 3C-D). Expression of wild type ckr-1 restored normal reorientation behavior when expressed under control of native $c k r-1$ promoter elements (3.5 kb) in $c k r-1$ (If) animals (Fig. 3C), but not when expressed under the ckr-2 promoter (Fig. S5B). Expression of ckr-1, but not ckr-2, also rescued reorientations in $c k r-1$ (If); $c k r-2$ (If) double mutants (Fig. S5A). These findings show that $n / p-12$ and $c k r-$ 1 act in the same genetic pathway and point to a selective requirement for NLP-12 signaling through CKR-1 in regulating trajectory changes during local searching. Deletion of $n / p-12$ did not produce significant changes in dispersal behavior, but we noted a modest decrease in reorientations during dispersal in ckr-1 mutants (Fig. 3E). This may indicate additional roles for CKR-1 during dispersal. Together, our genetic and behavioral studies implicate CKR-1 and CKR-2 GPCRs as targets of NLP12 signaling under conditions of overexpression and during basal locomotion. In contrast, we find that NLP-12 modulation of local searching is primarily achieved through CKR-1 activation. 


\section{Acute stimulation of DVA promotes reorientation behavior and requires NLP-12 and CKR-1}

We next addressed the question of how neuronal release of NLP-12 promotes area restricted searching. We measured trajectory changes elicited by acute depolarization of the DVA neuron. We used the $n / p-12$ promoter to drive cell-specific expression of Channelrhodopsin-2 (ChR2) ${ }^{32}$ in DVA and tracked worm movement during a 1 minute period of blue light $(470 \mathrm{~nm})$ photostimulation. We found that animals reorient more frequently with depolarization of DVA compared to pre-stimulus control (Fig. 3F). Importantly, light exposure did not increase reorientations in the absence of retinal (-ATR) (Fig. 3F). Depolarization of the DVA neuron in $n / p-12$ mutants failed to produce a similar enhancement (Fig. 3F), offering support for the idea that reorientations primarily arise due to release of NLP-12 peptides. Single $c k r-1$ deletion or combined $c k r-1$ and $c k r-2$ deletion also abrogated DVA-elicited increases in reorientation behavior, while single ckr-2 deletion produced more variable responses that were not clearly distinguishable from control (Fig. 3F). Our photostimulation experiments provide direct evidence that NLP-12 release from the DVA neuron promotes reorientation behavior, and, in addition, provide evidence for central involvement of NLP-12 signaling through the CKR-1 GPCR in directing reorientations. While NLP-12 expression has also been recently reported in PVD neurons ${ }^{33}$, expression of $n / p-12$ under a PVD specific promoter (ser-2prom3) did not restore reorientations in $n / p$ 12(If) animals (Fig.S5C), pointing towards DVA as the primary source of NLP-12 in promoting reorientations.

\section{Elevated CKR-1 signaling enhances turning and body bending in a nlp-12 dependent manner}

To further define the role of CKR-1, we next asked whether increased CKR-1 signaling would be sufficient to induce local search-like behavior. To address this question, we pursued an overexpression strategy similar to our above approach for $n l p-12$. We generated transgenic lines where the $c k r-1$ genomic sequence including native $c k r-1$ promoter elements was injected into wild type animals at high concentration. We found that $c k r-1$ overexpression produced striking increases in turning and large head to tail body bends (Fig. 4A, 6C, Video S4), qualitatively similar to the effects of 
nlp-12 overexpression (Fig. 1A). ckr-1(OE) animals made steep bends during runs of forward movement, with angles approaching $200^{\circ}$, whereas bending angles in wild type rarely exceeded $75^{\circ}$ (Fig. 4B). Notably, these high angle bends often produced spontaneous reorientations during forward movement and sometimes elicited sustained coiling. The amplitude of body bends during movement also increased by approximately 3-fold in $c k r-1(\mathrm{OE})$ animals compared to wild type (Fig. 4C). These increases in bending angles and body bend depth were returned to wild type levels by $n / p-12$ deletion (Fig. 4A-C), demonstrating that NLP-12 peptides are the major CKR-1 ligands required to elicit these characteristic changes in movement. Together, our genetic studies define NLP-12/CKR-1 as a novel ligand-GPCR pathway that controls trajectory changes and body bending to produce adaptive behavior.

\section{$c k r-1$ is expressed in many neurons that do not receive direct synaptic inputs from DVA}

To identify cells where CKR-1 may act to promote local searching, we generated strains expressing a $c k r-1$ reporter transgene that included the complete $c k r-1$ genomic locus and $\sim 3.5 \mathrm{~kb}$ of upstream regulatory sequence SL2 trans-spliced to sequence encoding GFP (green fluorescent protein) or mCherry. We found that $c k r-1$ is broadly expressed in the nervous system, showing expression in a subset of ventral nerve cord motor neurons, amphid and phasmid sensory neurons, premotor interneurons, and motor neurons in the nerve ring (Fig. 5A-B). We identified many of these neurons, largely from analysis of $c k r-1$ co-expression with previously characterized reporters (Table S2). Notably, ckr-1 and ckr-2 expression showed little overlap (Fig. S7).

In the ventral nerve cord, we found that $c k r-1$ is expressed in cholinergic, but not GABAergic, ventral cord motor neurons (Fig. S6A-B, Table S2). Amongst head neurons, the $c k r-1$ reporter is expressed in GABAergic RMEV, RMED, AVL and RIS neurons, cholinergic SMDV, SMDD and RIV head motor neurons, the interneuron RIG, the serotonergic NSM neuron, and in the interneurons AIA and AIB (Fig. $5 \mathrm{~B}$, Table S2). Additional studies using Dil uptake indicated that $c k r-1$ is also expressed in the amphid 
sensory neurons ASK and ASI and the phasmid sensory neurons PHA and PHB (Table S2). With the exception of the ventral cord cholinergic neurons, the ckr-1 reporter almost exclusively labeled neurons that do not receive direct synaptic input from DVA, suggesting that NLP-12 acts at least partially through extrasynaptic mechanisms.

\section{CKR-1 functions in the SMD head motor neurons to modulate body bending}

We next pursued cell-specific $c k r-1$ overexpression to gain insight into which of the $c k r$-1-expressing neurons defined above may be primary targets for modulation during local searching (Table S3-S4). We focused our analysis on body bending amplitude because this was the most easily quantifiable aspect of movement to be modified by $c k r-1$ overexpression. Transgenic strains where pan-neuronally expressed ckr-1 (rgef-1 promoter) was injected at high concentration displayed increased body bending amplitude, similar degree to overexpression using the native promoter (Fig. 5C). In contrast, ectopic ckr-1 expression in muscles produced no appreciable change, consistent with a primary site of CKR-1 action in neurons (Fig. 5C). Surprisingly, ckr-1 overexpression in cholinergic ventral cord motor neurons (unc-17 $\beta$ promoter) also did not elicit an appreciable change in body bend depth (Fig. 5C). We therefore next targeted the head neurons identified by our ckr-1 reporter, using several different promoters for ckr-1 overexpression in subsets of head neurons (Fig. 5C, Table S3-S4). ckr-1 overexpression using either the odr-2(16) or lgc-55 promoters produced a striking (2.5-fold) increase in body bend depth, comparable with ckr-1 overexpressed under its endogenous promoter. In contrast, ckr-1 overexpression in GABAergic neurons, including RMED and RMEV (using unc-47 promoter), did not produce an appreciable effect. Likewise, ckr-1 overexpression in RIV, RIG, NSM, AIA, AIB or amphid neurons failed to significantly enhance body bend depth. The lgc-55 promoter drives expression in AVB, RMD, SMD and IL1 neurons, as well as neck muscles and a few other head neurons ${ }^{34}$, while the odr-2(16) promoter primarily labels the RME and SMD head neurons ${ }^{35}$ (Table S2S3). The overlapping expression of the odr-2(16) and lgc-55 promoters in SMD neurons suggested that these neurons may be centrally involved. SMD co-labeling by $c k r-1:: S L 2:: m$ Cherry and Plad- 
2::GFP36 provided additional evidence for $c k r-1$ expression in these neurons (Fig. S6C). Intriguingly, we noted that NLP-12::Venus clusters are concentrated in the nerve ring region of the DVA process (Fig. 5D), in the vicinity of SMD processes (Fig. 5E). In contrast to ckr-1, ckr-2 was either absent or more variably expressed in a subset of the SMD neurons, the SMDDs (Fig. S6D) ${ }^{37}$.

The 4 SMDs (dorsal-projecting SMDDL and SMDDR and ventral-projecting SMDVL and SMDVR) are bilateral motor neuron pairs that innervate dorsal and ventral head/neck musculature, and also form reciprocal connections with one another. ${ }^{28}$ They have been previously implicated in directional head bending and steering. ${ }^{38-41}$ To better define the behavioral effects of SMD modulation, we more closely examined body bending in animals overexpressing $c k r-1$ under control of the odr-2(16) promoter, and also using a second promoter, flp-22 $\Delta 4$, that was recently shown to drive selective expression in the SMD neurons $^{42}$ (Fig. 6A). For both overexpression strains, we observed significant increases in body bending amplitude and bending angle compared to wild type (Fig. 5C, 6B-C, Video S5). These increases were dependent on NLP-12 signaling (Fig. 6C, S8A-B) and were similar to those observed for native $c k r-1$ (Fig. 4, 6C) and nlp-12 overexpression (Fig. 1, 6C). Thus, the actions of CKR-1 in the SMD motor neurons recapitulate many of the behavioral effects of NLP-12 overexpression.

To ask if the SMD neurons are required for the locomotor changes produced by $c k r-1$ overexpression, we expressed the photoactivatable cell ablation agent PH-miniSOG in the SMD neurons (Pflp-22 $\Delta 4)$ of animals overexpressing $c k r-1$ (native promoter). When activated by blue light $(470 \mathrm{~nm}) \mathrm{PH}-\mathrm{miniSOG}$ produces reactive oxygen species and disrupts cellular function. ${ }^{43}$ Following photoactivation of miniSOG in animals overexpressing $c k r-1$, we observed striking decreases in bending angles (Fig. 6DE) and amplitude (Fig. 6F) during movement. We confirmed successful SMD ablation by examining morphological changes in GFP-labeled SMD neurons following photoactivation of miniSOG (Fig. 6D). Expression of miniSOG did not have appreciable effects on the body bending of $c k r-1(O E)$ animals 
under control conditions (without light exposure) (Fig. S8C). In addition, stimulation of control animals without the miniSOG transgene did not appreciably alter body bending (Fig. 6E) or SMD neuron morphology (Fig. S8D). These results indicate that SMD motor neurons are required for the locomotor effects of $c k r-1$ overexpression, and, importantly, raise the possibility that the SMD neurons are key targets for NLP-12 neuromodulation during local searching in wild type.

\section{NLP-12/CKR-1 excitation of the SMD neurons promotes local searching}

To further investigate the site of CKR-1 function, we examined rescue of area restricted searching in ckr-1 mutants by generating additional transgenic lines providing for SMD-specific expression of wild type $c k r-1$ (injected at 5-fold lower concentration than used for overexpression above). Injection of wild type animals with the SMD::ckr-1 transgene at this lower concentration did not appreciably increase bending depth or angle (Fig. S5D). However, expression in ckr-1 mutants restored reorientations during food searching to roughly wild type levels (Fig. 7A), indicating that CKR-1 function in the SMD neurons is sufficient to support NLP-12 modulation of local searching.

To investigate how increased SMD activity may impact movement, we photostimulated the SMDs in animals expressing Podr-2(16)::Chrimson. ${ }^{44}$ Prior to photostimulation, animals demonstrated long forward runs with relatively few changes in trajectory (Fig. 7B). Following the onset of photostimulation, Chrimson-expressing animals rapidly increased reorientations (Fig. 7B-C, Video S6), while control animals (-Retinal) did not increase trajectory changes during the light stimulation period (Fig. 7C). SMD photostimulation also elicited a modest increase in body bending (Fig. S8E).

Conversely, transient and inducible silencing of the SMDs by histamine-gated chloride channel expression significantly reduced reorientations during food searching (Fig. 7D). Thus, direct activation or inhibition of SMD neurons alter turning and reorientations, consistent with a potential mechanism for NLP-12/CKR-1 modulation of local searching through signaling onto the SMD neurons. 
To explore the dynamics of SMD neuronal activity during searching, we next measured calcium responses from SMD neurons of behaving animals. We simultaneously recorded GCaMP6s and mCherry fluorescence (flp-22 $\Delta 4$ promoter) continuously from behaving animals during ARS (0-5 minutes off food) and dispersal (30-35 minutes off food). We observed elevated SMD activity in wild type animals during ARS, compared with dispersal (Fig. 7E-F). By contrast, SMD activity during ARS was strikingly reduced in $c k r-1$ (If) animals compared to wild type (Fig. 7E-F), supporting a model (Fig. 8) where NLP-12/CKR-1 signaling promotes local searching by increasing the activity of SMD head motor neurons.

\section{Discussion}

Neuropeptidergic systems have crucial roles in modulating neuronal function to shape alternate behavioral responses, but we have limited knowledge of the circuit-level mechanisms by which these alternate responses are generated. Here, we show that the C. elegans NLP-12 neuropeptide system, closely related to the CCK system in mammals, shapes adaptive behavior through modulation of motor circuits dedicated to control of either head or body wall musculature. We demonstrate that NLP-12 modulation of these circuits occurs through distinct GPCRs, CKR-1 and CKR-2, that primarily act on either head or body wall motor neurons respectively. Under basal conditions, we suggest that NLP-12 modulation of the body wall motor circuit predominates, influencing the depth of body bends during sinusoidal movement through CKR-1 and CKR-2 GPCRs located on body wall motor neurons. NLP-12 activation of head motor neurons through CKR-1 becomes predominant in the absence of food, promoting reorientations. We propose that changes in food availability reconfigure functional connectivity in the NLP-12 system by differentially engaging GPCRs across the head and body wall motor circuits. Intriguingly, the involvement of 2 GPCRs in nematode NLP-12 signaling is reminiscent of the organization of the CCK system in mammals, which relies on signaling through CCK1 and CCK2 GPCRs. CCK is among the most abundant peptides in the mammalian brain. New details 
about CCK signaling in the brain and the GPCRs involved are continuing to emerge. ${ }^{12-15,45-48}$ Our findings may point towards similar utilization of specific CCK-responsive GPCRs to coordinate activity across circuits in mammals.

NLP-12 neuropeptides act as key modulators in a range of $C$. elegans behaviors. Local search responses to varying oxygen levels and decreased food availability both involve NLP-12 signaling. ${ }^{6,7}$ Additionally, NLP-12 signaling has been implicated in various aspects of proprioceptive signaling and postural control. ${ }^{29,30}$ However, the mechanisms by which NLP-12 peptides exert their influence over these diverse behavioral responses have remained unclear. Our work addresses these mechanistic questions by defining roles for CKR-1 and CKR-2 GPCRs during basal locomotion and area-restricted searching. Area-restricted searching is a complex motor behavior, involving rapid trajectory changes that serve to maintain the animal within a restricted area of their immediate environment.6,7,24,27 Reorientations during searching are produced through high angle forward turns ${ }^{6,49}$ and reversal-coupled omega turns $\mathbf{s}^{6,24}$. We previously demonstrated a requirement for NLP-12 in promoting reorientations during local searching. ${ }^{6}$ Our analysis here shows that loss of $n / p-12$ also has modest effects on body posture during normal exploratory movement, indicating NLP-12 regulation of motor targets under basal conditions. Intriguingly, the behavioral requirement for NLP-12 is far more apparent during local searching compared with basal locomotion, suggesting enhanced involvement of NLP-12 signaling for performance of local searching. Similar observations about NLP-12 involvement in chemotactic responses to varying oxygen levels suggested a model for graded NLP-12 regulation of movement. ${ }^{7}$ Based on our observations, we speculate that increased engagement of head motor neurons through CKR-1 activation may be a generalizable mechanism for dynamic NLP-12 regulation of behavior over changing external conditions. 
Prior studies had implicated the CKR-2 GPCR in NLP-12 function $9,29,30$, but roles for CKR-1 had not been previously described. CKR-2 shows slightly broader expression compared with CKR-1, but both GPCRs are expressed across a variety of neuron classes, including many that do not receive direct synaptic inputs from DVA. We noted very little overlap in CKR-1 and CKR-2 expression, consistent with the idea that the two GPCRs serve distinct roles in modulating behavior. Our genetic analyses and heterologous expression studies firmly establish CKR-1 as a functional target for NLP-12 signaling with an activation profile similar to CKR-2. NLP-12 activation of CKR-2 stimulates neurotransmission through coupling with egl-30 $\left(\mathrm{G}_{\alpha \mathrm{q}}\right)$ and egl-8 (PLC $\beta$ ) likely by DAG interaction with the synaptic vesicle priming factor UNC-13.29,30 Given the sequence homology between CKR-1 and CKR-2, it seems likely that CKR-1 also functions to positively regulate neuronal activity through egl-30. In support of this idea, we found that SMDspecific CKR-1 overexpression and SMD neuron photostimulation produced qualitatively similar behavioral effects, an increase in reorientations during movement. We also noted elevated $\mathrm{Ca}^{2+}$ activity in SMD neurons during ARS that was strongly dependent upon ckr-1 expression. The DVA neuron makes a single synapse with SMDVL (Worm wiring). While it is possible that this single synapse accounts for NLP-12 elicited behavioral changes during local searching, it seems likely that extrasynaptic signaling to other SMD neurons also contributes.

SMDs innervate head and neck muscles ${ }^{28,50}$ and biased activity of dorsal or ventral SMDs is correlated with directional head bending38,39,41,42,51. Turning is preceded by head bending or head swings. Shallow bends promote more gradual turns, while large head swings drive sharp turns. ${ }^{49,52}$ We found that SMD activity is elevated during wild type local searching compared with dispersal, and reduced by deletion of $c k r-1$. Our results suggest NLP-12 activation of CKR1 may modulate functional connectivity between SMD neurons and dorsal and ventral head/neck muscles. We speculate that elevated SMD activity is permissive for reorientations to 
occur. According to this model, enhanced transmission from dorsal or ventral SMD synaptic outputs onto head and neck muscles would bias toward exaggerated head bending, thereby promoting periods of enhanced turning and trajectory changes. CKR-1 activation might also influence SMD proprioceptive functions ${ }^{42}$ or functional connectivity with other neurons implicated in regulation of head posture and bending, such as RIA ${ }^{38,51}$ and RME ${ }^{39}$.

Surprisingly, selective $c k r-1$ overexpression using the odr-2(16) or flp-22 4 promoters increased body bend depth, raising the question of how altered SMD activity might translate into increased body bending. Recent work suggests an interesting functional coupling between the activity of SMD neurons and ventral cord B-type motor neurons. ${ }^{41}$ B-type motor neurons are suggested to act as a distributed central pattern generator for the propagation of body bends. ${ }^{53,54} \mathrm{CKR}-1$ activation of SMDs may therefore influence body depth directly by altering body wall motor neuron excitability through a gap junction connection between VB1 and SMDVR or through neuromuscular synapses located in the sublateral processes.

The similar potency of NLP-12 peptides for activating CKR-1 and CKR-2, suggests that differential contributions of these GPCRs during basal locomotion and search responses do not arise due to dramatic differences in NLP-12 potency to activate each receptor. This raises important questions about how a bias toward CKR-1 modulation of the head motor circuit during local searching may occur. We envision that NLP-12 regulation of the SMD neurons acts in parallel with other neural pathways previously shown to promote reversals during local searching. For example, olfactory information about food availability is conveyed by sensory neurons such as AWC and ASK to premotor interneurons (AIA, AIB, AIY) and ultimately transformed into patterns of motor neuron activity that drive reversals. ${ }^{24,25,51,55}$ The SMD neurons also receive synaptic information from this circuit (for example, through synaptic 
connections from the AIB and RIM neurons) ${ }^{28}$, raising the possibility that a pathway activated by food removal may enhance SMD sensitivity to CKR-1 activation. In this case, SMD neurons may be a site for integration of information encoding reversals and forward reorientations during local searching. A shift to CKR-1 modulation of head neurons during searching could also be triggered by dopaminergic stimulation of DVA. Prior work implicated dopaminergic signaling from PDE neurons in the regulation of NLP-12 and motor responses. ${ }^{6,8}$ In this case, elevated levels of NLP-12 secretion, perhaps from release sites in the nerve ring region, would be predicted to bias the system towards enhanced activation of the SMD neurons and elicit increased turning. Notably, PDE also regulates an antagonistic peptidergic circuit, mediated by FLP-1 neuropeptides, through inhibitory connections with AVK interneurons ${ }^{8}$, suggesting the potential for more complex behavioral regulation.

Our studies of the nematode NLP-12 system offer new mechanistic insights into neuropeptide modulation of behavior. Our findings provide a key first step in defining roles for two NLP-12responsive GPCRs in coordinating motor control across changing conditions. We propose that the NLP-12 system conditionally engages GPCRs expressed in head or body motor neurons to modify specific features of locomotion, most notably reorientations during searching and body bend depth during basal locomotion. Brain CCK has been increasingly implicated as a key regulator in diverse aspects of behavior, including feeding, satiety, memory, nociception and anxiety. ${ }^{14,45,56-59}$ Thus our studies elucidating mechanisms for NLP-12 regulation of circuit function in the compact nematode nervous system may have important and broadly applicable implications for neuromodulation in more complex systems. 


\section{Materials and Methods}

\section{Strains}

All nematode strains (Table S1) were maintained on OP50 seeded agar nematode growth media (NGM) at room temperature $\left(22-24^{\circ} \mathrm{C}\right)$. N2 Bristol strain was used as wild type.

Transgenic animals were generated by microinjection into the germ line and transformation monitored by co-injection markers. Multiple independent extrachromosomal lines were obtained for each transgenic strain and data presented from a single representative transgenic line.

Stably integrated lines were generated by X-ray integration and outcrossed at least four times to wild type.

\section{Molecular Biology}

All plasmids, unless specified, were generated by Gateway cloning (see Supplementary Tables). p-ENTR plasmids were generated for all promoters used (Table S5). The ckr-1 minigene construct (pRB12/pRB13) was generated by cloning the $c k r-1$ coding sequence (start to stop), with introns 1, 8 and 9 . For cell specific overexpression or rescue, the $c k r-1$ minigene was recombined with entry vectors containing the relevant cell-specific promoters. (Table S3S4).

Behavioral assays and analyses

All behavioral assays were carried out using staged 1-day adult animals on Bacto-agar NGM agar plates seeded with a thin lawn of OP50 bacteria $(50 \mu \mathrm{L})$ unless otherwise noted. Video recordings for behavioral analyses were obtained using a Firewire camera (Imaging Source) and ICCapture2.2. Animals were allowed to acclimate for 30 seconds prior to video recording. Post hoc locomotor analysis was performed using Wormlab (MBF Bioscience) (Video S8). Videos were thresholded to detect worms, and worm movement was tracked. Body bend amplitude was quantified as the average centroid displacement over the duration of a locomotion track (Fig. 1B). Body bending angle was measured, at the midbody vertex, as the supplement of the angle between the head, mid-body, and tail vertices (Fig. 1C). Bending 
angles were measured, continuously for each frame tracked, over 30 s (900 frames @ 30 fps). The measured bending angles were binned to generate a frequency distribution of body bending angles. Kymographs were generated from worm body curvature data (Wormlab) using custom MATLAB script.

\section{Area restricted search behavior}

For quantification of local search behavior, single well-fed animals were transferred to an intermediate unseeded plate. After one minute, animals were repicked without bacteria and transferred to an unseeded behavior assay plate. Digital movies were captured over the first 5 mins (local search) and after 30 mins (dispersal) following removal from food. Reorientations were manually scored post hoc from monitoring movement direction, over sequential frames ( 200 frames for forward reorientations, 600 frames for reversal-coupled omega turns) from the start of the reorientation (original trajectory) to when the animal completed the reorientation (new trajectory) (Fig. 3B, S3). A forward reorientation was scored after animals moved a minimum of 3 s ( 100 Frames @ 30 fps) along a new trajectory. We scored forward trajectory changes $>50^{\circ}$ and reversal coupled omega turns as reorientations (examples of each in Fig.3B, S3). Trajectory changes where animals initially performed head bends $>50^{\circ}$, but then resumed the original path of movement or altered immediate trajectory $<50^{\circ}$ were not scored as reorientations. Trajectory changes were quantified (in degrees) using the angle tool (ImageJ) to measure the angle between the original and new trajectory (Fig. 3B, S3). We excluded reversals and post reversal changes in trajectory that did not involve omega turns.

\section{Single worm tracking}

Single worm tracking was carried out using Worm Tracker $2 .{ }^{60}$ Animals were allowed to acclimate for 30 seconds prior to tracking. Movement features were extracted from five minutes of continuous locomotion tracking (Video S7). Worm tracker software version 2.0.3.1, created by Eviatar Yemini and Tadas Jucikas (Schafer lab, MRC, Cambridge, UK), was used to analyze 
movement. ${ }^{61}$ Worms were segmented into head, neck, midbody, hips and tail. The body bend angle is angle measured at the midbody vertex, between the neck and hip skeleton vertices (Fig. 2A). Head bend angles were measured as the largest bend angle prior to returning to a straight, unbent position (Fig. 2B). Absolute midbody bending (Fig. 2A) and head bending (Fig. 2B) angles were quantified. Single worm tracking affords higher resolution and allows for rich quantification of relatively subtle postural changes. However, continuous tracking of animals was difficult to achieve using this approach during the numerous steep turns performed during ARS, or with NLP-12 or CKR-1 overexpression. Post hoc analysis of videos to measure body bending (as described earlier) proved most reliable.

SMD ablation

Cell ablation protocol by miniSOG activation was adapted from Xu et. al. 2016. ${ }^{43}$ MiniSOG activation was achieved by stimulation with repetitive $2 \mathrm{~Hz} 250 \mathrm{~ms}$ blue light pulses for 12 minutes [200mW/cm², $488 \mathrm{~nm} 50 \mathrm{~W}$ LED (Mightex Systems)]. Experiments were performed on unseeded plates using larval stage $4 c k r-1(O E)$ animals expressing miniSOG and GFP transgenes under the flp-22 44 promoter. Following stimulation, animals were allowed to recover in the dark on NGM OP50 plates for 16 hours prior to behavioral analysis or imaging.

Photostimulation experiments

All-Trans Retinal (ATR) plates were prepared (100 mM stock in ethanol, final working $2.7 \mathrm{mM}$ in OP50). Plates were stored at $4^{\circ} \mathrm{C}$ under dark conditions and used within one week. Animals were grown on +ATR OP50 plates in dark and L4 animals were transferred to a fresh +ATR plate prior to the day of experiment. Experiments were performed using one-day adults. For ChR2 photostimulation, experiments were conducted using a fluorescent dissecting microscope (Zeiss stereo Discovery.V12) equipped with a GFP filter set. Behavior was recorded for a 1minute period prior to photostimulation and during a subsequent 1-minute period during photostimulation. Data are expressed as \% change in reorientations across these time intervals. 
Chrimson photostimulation $\left(26 \mathrm{~mW} / \mathrm{cm}^{2}\right)$ experiments were conducted using a $625 \mathrm{~nm} 50 \mathrm{~W}$ LED (Mightex Systems). Animals were video recorded for 1 minute in the absence of light stimulation (prestimulus) and subsequently for 1 minute with light stimulation. Control experiments (-ATR) were performed in the same manner.

SMD silencing

ARS assays were performed on unseeded Histamine $(10 \mathrm{mM})$ and control Bacto-agar NGM plates using staged 1 day adults. For SMD silencing, transgenic animals were placed on Histamine plates, seeded with $100 \mu \mathrm{L}$ OP-50, for 1 hour prior to experiment. ARS was quantified as described previously.

Imaging

Fluorescent images were acquired using either BX51WI (Olympus) or Yokogawa (Perkin Elmer) spinning disc confocal microscopes. Data acquisition was performed using Volocity software. Staged one-day adult animals were immobilized using $0.3 \mathrm{M}$ sodium azide on $2 \%$ agarose pads. Images were analyzed using ImageJ software.

\section{SMD calcium imaging}

Calcium imaging was performed in behaving transgenic animals, expressing GCaMP6s::SL2::mCherry under flp-22 44 promoter, on $5 \%$ agarose pads on a glass slide. Animals were treated as described for ARS and dispersal assays, and simultaneous dualchannel (GCaMP6s and mCherry) recordings were performed in the time windows of ARS (0-5 minutes) and dispersal (30-35 minutes off food). Imaging was carried out on an Axio Observer A1 inverted microscope (Zeiss) connected to a Sola SE Light Engine (Lumencor) with a Olympus 2.5X air objective, and a Hamamatsu Orca-Flash 4.0 sCMOS camera. Simultaneous GCaMP and mCherry acquisition was achieved using the optical splitter Optisplit-II (Cairn Research) with filters ET525/50M and ET632/60M, and dichroic T560Iprx-UF2 (Chroma). Image acquisition was performed using Micromanager, at 66 ms exposure (approximately $15 \mathrm{fps}$ ).

ROIs encompassed cell bodies in the nerve ring labeled by mCherry and were tracked using a 
custom Matlab script (Neuron Activity Analysis, Mei Zhen, Video S9). Frames where movement artifacts were encountered due to stage movement were not included in analysis. The

\section{background subtracted calcium signals were plotted as a ratio (GCaMP6s/mCherry).}

in vitro GPCR characterization

The GPCR activation assay was performed as previously described. ${ }^{31,62,63}$ Briefly, CHO-K1 cells stably expressing the luminescent $\mathrm{Ca}^{2+}$ indicator aequorin and the promiscuous $\mathrm{G}_{\alpha 16}$ protein (ES-000-A24 cell line, PerkinEImer) were transiently transfected with $c k r-1 / p c D N A 3.1$, ckr-2/pcDNA3.1 or empty pcDNA3.1 vector. Cells were transfected with Lipofectamine LTX and Plus reagent (Invitrogen) at $60-80 \%$ confluency and grown overnight at $37^{\circ} \mathrm{C}$. After 24 hours, they were shifted to $28^{\circ} \mathrm{C}$ overnight. On the day of the assay, transfected cells were collected in bovine serum albumin (BSA) medium (DMEM/F12 without phenol red with L-glutamine and 15 mM HEPES, Gibco, supplemented with $0.1 \%$ BSA), at a density of 5 million cells per $\mathrm{mL}$, and loaded with $5 \mu \mathrm{M}$ coelenterazine h (Invitrogen) for 4 hours at room temperature. Compound plates containing synthetic peptides in DMEM/BSA were placed in a MicroBeta LumiJet luminometer (PerkinElmer). After loading, the transfected cells were added at a density of 25,000 cells/well, and luminescence was measured for 30 seconds at a wavelength of $469 \mathrm{~nm}$. After 30 seconds, $0.1 \%$ triton $\mathrm{X}-100$ (Merck) was added to lyse the cells, resulting in a maximal $\mathrm{Ca}^{2+}$ response that was measured for 30 seconds. To constitute concentration-response curves of NLP-12 peptides, peptide concentrations ranging from $1 \mathrm{pM}$ to $10 \mu \mathrm{M}$ were tested in triplicate on two independent days. 

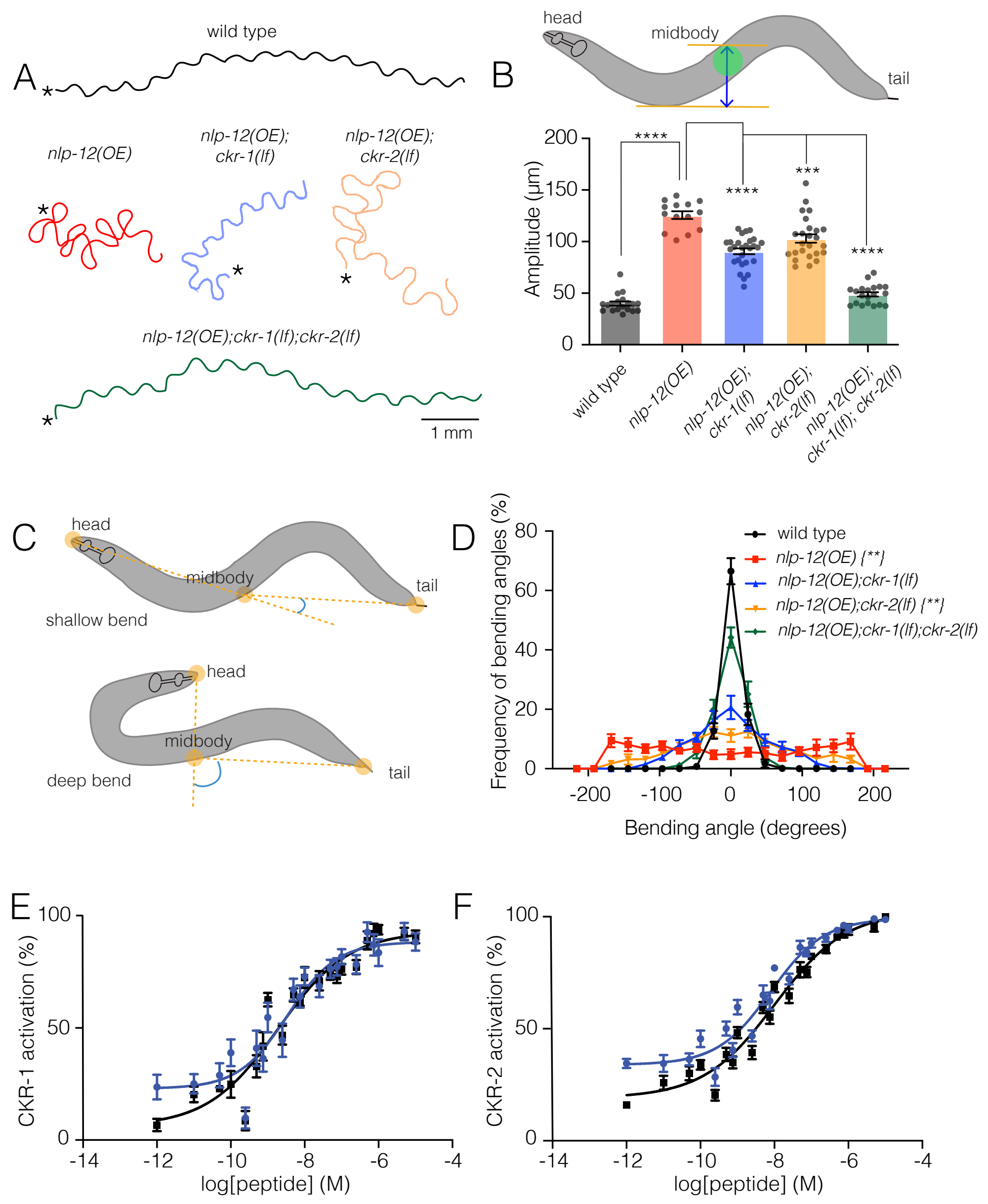

$\rightarrow$ NLP-12-1: DYRPLQFamide $\mathrm{EC}_{50}=3.5 \mathrm{nM}$

$\rightarrow$ NLP-12-2: DGYRPLQFamide $\mathrm{EC}_{50}=1.9 \mathrm{nM}$

$\rightarrow$ NLP-12-1: DYRPLQFamide $\mathrm{EC}_{50}=8.0 \mathrm{nM}$

$\rightarrow-$ NLP-12-2: DGYRPLQFamide $\mathrm{EC}_{50}=10.2 \mathrm{nM}$ 


\section{FIGURE LEGENDS}

\section{Figure 1. NLP-12/CCK induced locomotor responses require functional $c k r-1$ signaling}

(A) Representative movement trajectories of wild type (black), nlp-12(OE) (red), nlp-12(OE);ckr-1(If) (blue), nlp-12(OE);ckr-2(If) (orange) and nlp-12(OE);ckr-1(If);ckr-2(If) (green) animals during forward runs (30s) on NGM agar plates seeded with OP50 bacteria. $n / p-12(O E)$ refers to the transgenic strain (ufls104) stably expressing high levels of wild type $n / p-12$ genomic sequence. Note the convoluted $n / p-$ 12(OE) movement tracks are restored to wild type by combined $c k r-1$ and $c k r-2$ deletion. Scale bar, 1 $\mathrm{mm}$. Asterisks $\left(^{*}\right)$ indicate position of worm at start of recording.

(B) Average body bend amplitude (indicated in schematic by blue arrow between orange lines, midbody centroid (green) of worm) for the genotypes as indicated. Bars represent mean \pm SEM. In this and subsequent figures. ${ }^{* * *} p<0.0001,{ }^{* * *} p<0.001$, ANOVA with Holms-Sidak post-hoc test. wild type $\mathrm{n}=19, n / p-12(O E): \mathrm{n}=14, n / p-12(O E) ; c k r-1(I f): \mathrm{n}=27, n l p-12(O E) ; c k r-2(I f): \mathrm{n}=25, n l p-12(O E) ; c k r-1(I f) ; c k r-$ 2(lf): $n=20$

\section{(C) Schematic representation of measured body bending angle, for shallow (top) and deep (bottom)}

body bends. Solid orange circles indicate the vertices (head, midbody and tail) of the body bending angle (blue) measured.

(D) Frequency distribution of body bending angle (indicated in blue in 1C) for the genotypes indicated. Kolmogorov-Smirnov test: wild type vs n/p-12(OE) **, wild type vs n/p-12(OE);ckr-2(If) **, nlp-12(OE) vs nlp-12(OE);ckr-1(If);ckr-2(If)**, ${ }^{* *} p<0.01$. wild type: $\mathrm{n}=12, n / p-12(O E): \mathrm{n}=10, n / p-12(O E) ; c k r-1$ (If): $\mathrm{n}=10, n / p-12(O E) ; c k r-2(I f): \mathrm{n}=12, n l p-12(\mathrm{OE}) ; c k r-1(I f) ; c k r-2(I f): \mathrm{n}=12$.

(E, F) Concentration-response curves of the mean calcium responses (\% activation \pm SEM) in CHO cells expressing either CKR-1 (E) or CKR-2 (F) for different concentrations of synthetic peptides NLP-12-1 (solid blue circles) or NLP-12-2 (solid black squares). Solid lines indicate curve fits to the data $(n=6) .95 \%$ confidence intervals $(n M)$, CKR-1: NLP-12-1, 1.79-7.07; NLP12-2, 0.93-3.77 and CKR-2: NLP-12-1, 5.16-12.51; NLP-12-2, 6.43-16.73. 

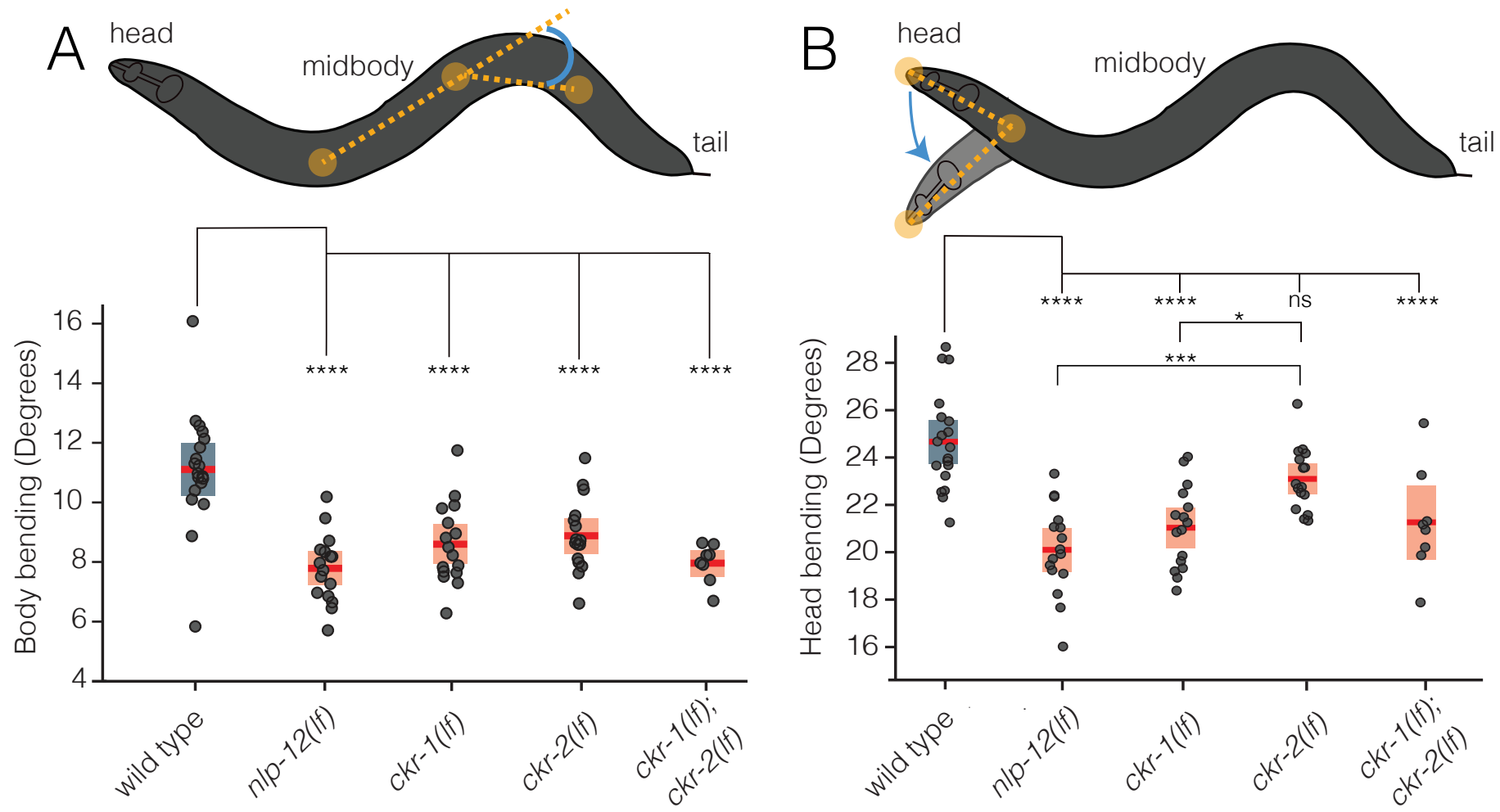


\section{Figure 2. CKR-1 and CKR-2 differentially regulate head and body bending during basal} locomotion

Schematics showing body bending $(\mathbf{A})$ and head bending $(\mathbf{B})$ angles (solid orange circles

indicate the vertices and measured angle in blue) quantified during single worm track analyses of movement (5 minutes) in the presence of food. Each data point in the scatterplots represents the average body or head bend angle for a single animal from analysis of 5 minutes of locomotion. Horizontal red bar indicates mean, shading indicates SEM for wildtype (blue) and mutants (orange). ${ }^{* * *} p<0.0001,{ }^{* * *} p<0.001,{ }^{*} p<0.05$, ns not significant. ANOVA with HolmsSidak post-hoc test. wild type: $n=19, n l p-12(o k 335): n=16, c k r-1(o k 2502): n=16, c k r-2(t m 3082)$ : $\mathrm{n}=16, c k r-1(0 k 2502) ; c k r-2(t m 3082): \mathrm{n}=8$. 
A

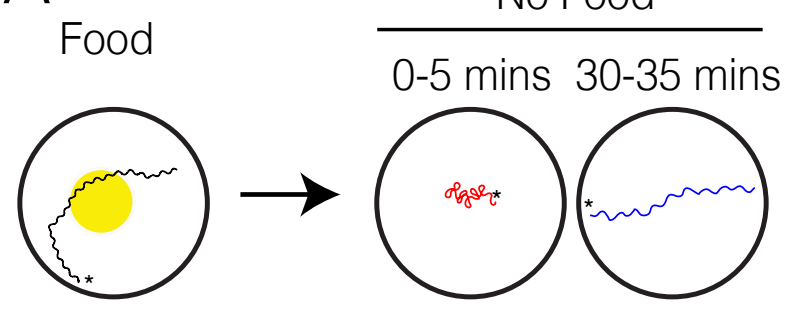

B
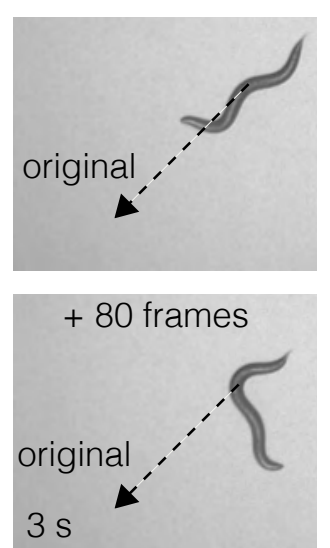

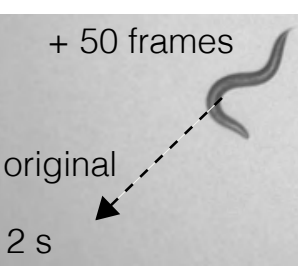

+130 frames

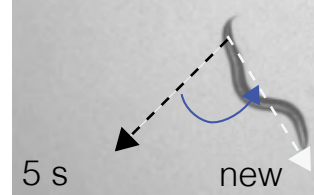

c

C
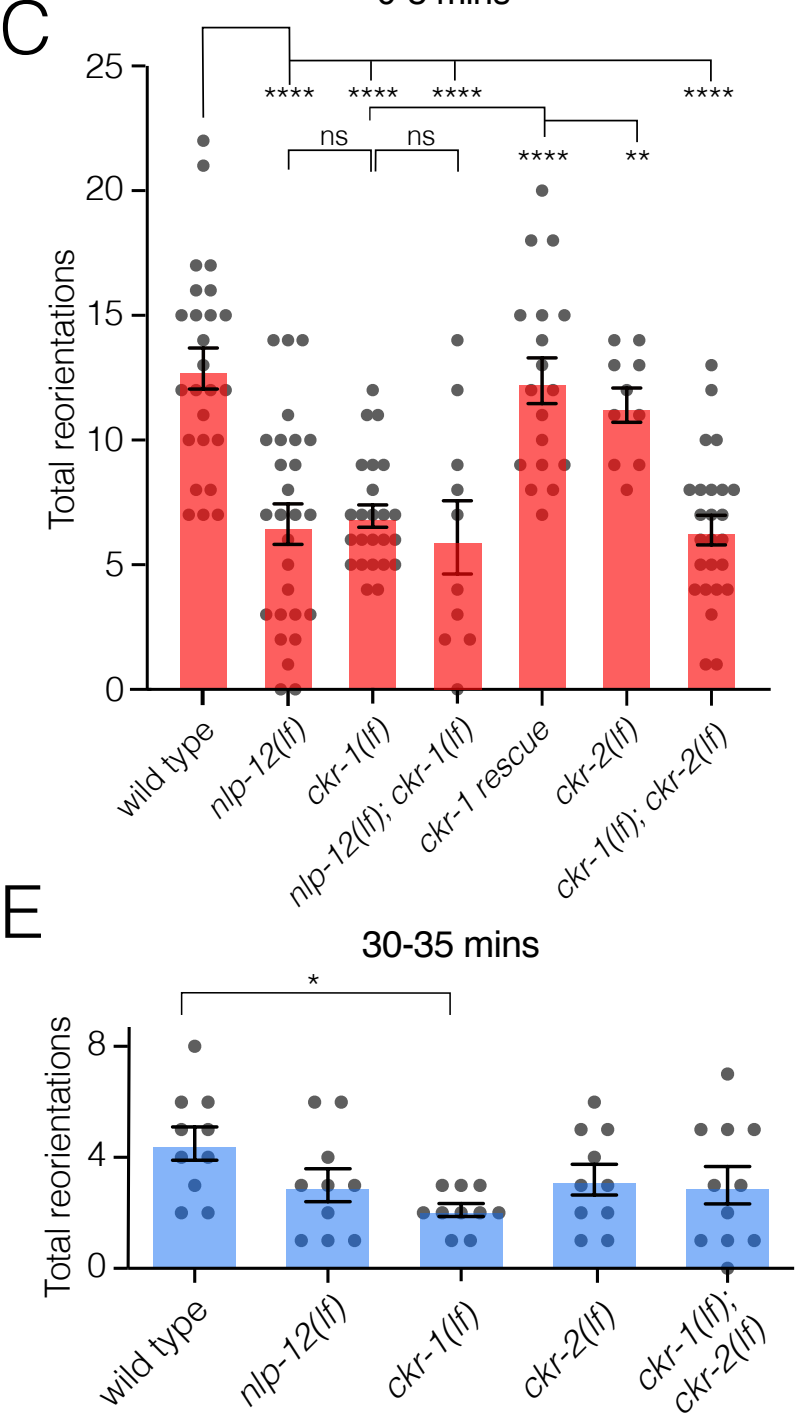

D
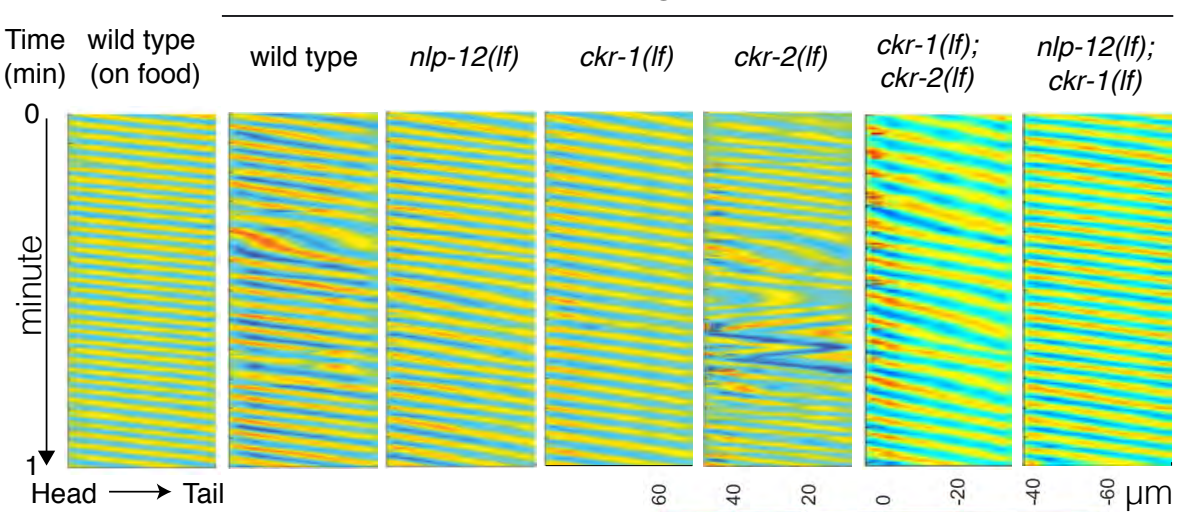

8 요

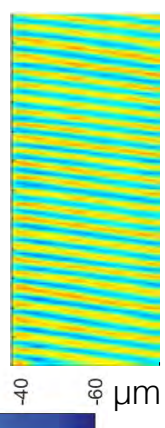

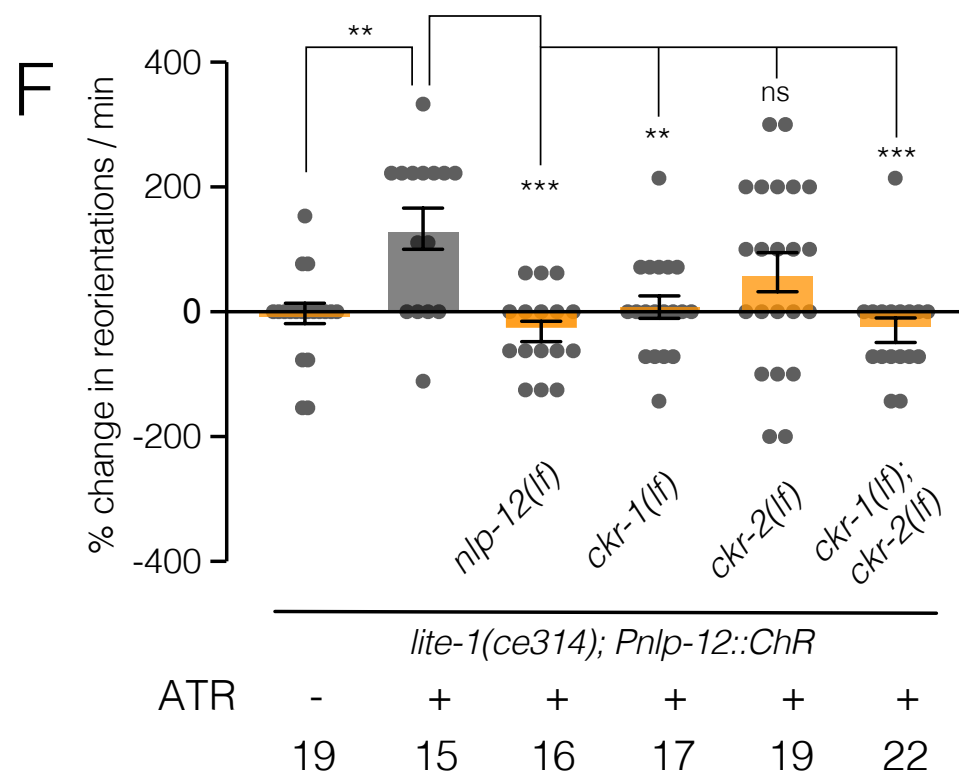


Figure 3. NLP-12/CCK food search responses are mediated through the GPCR CKR-1

(A) Schematic of the food search assay indicating the time intervals when reorientations were scored. Wild type animals increase reorientations during the first 5 mins (0-5 mins) after removal from food (local search) and reduce reorientations during dispersal (30-35 mins). Asterisks $\left(^{*}\right)$ indicate position of worm at start of recording.

(B) Frame grabs showing worm position and posture prior to, during and after reorientation. Angle (blue) between the black (original trajectory) and white (new trajectory) dashed lines indicates the change in trajectory. Frame numbers and time points indicated are relative to first image in each sequence, which represents the start point (frame 0 , time $0 \mathrm{~s}$ ) when the reorientation event began, and the last frame was when the reorientation was completed.

Trajectory changes were scored as reorientations if changes in trajectory were greater than $50^{\circ}$.

(C) Quantification of reorientations during 0-5 minutes following removal from food for the genotypes indicated. Rescue refers to transgenic expression of wild type $c k r-1$ in $c k r-1$ mutants. Bars represent mean \pm SEM. ${ }^{* * *} p<0.0001,{ }^{* *} p<0.01$, ns not significant, ANOVA with HolmsSidak's post-hoc test. wild type: $\mathrm{n}=25, \mathrm{nlp}-12$ (ok335): $\mathrm{n}=27, c k r-1$ (ok2502): $\mathrm{n}=24, n / p-$ 12(ok335);ckr-1(ok2502): $n=10, c k r-1$ rescue: $n=18, c k r-2(t m 3082): n=10, c k r-1$ (ok2502);ckr2(tm3082): $\mathrm{n}=25$.

(D) Representative body curvature kymographs for worm locomotion during basal locomotion and area restricted searching (ARS). Head to tail orientation along the horizontal axis in each kymograph is left to right as indicated for wild type. Time is indicated along the vertical axis from 0 to 1 minute. (E) Total number of reorientations during an interval of 30-35 minutes following removal from food for the genotypes as shown. Each bar represents mean \pm SEM. * $p<0.05$, ANOVA with Holms-Sidak's post-hoc test. wild type: $n=10, n / p-12(o k 335): n=10, c k r-1(o k 2502): n=10, c k r-$ 2(tm3082): $\mathrm{n}=10, c k r-1(0 k 2502) ; c k r-2(t m 3082): \mathrm{n}=11$.

(F) Trajectory changes (reorientations) scored in response to photostimulation of DVA. Percent change in the number of high angle turns elicited during $1 \mathrm{~min}$ of blue light exposure compared to 
bioRxiv preprint doi: https://doi.org/10.1101/2020.04.27.064550; this version posted June 28, 2021. The copyright holder for this preprint (which was not certified by peer review) is the author/funder. All rights reserved. No reuse allowed without permission.

prestimulus (no blue light). Bars represent mean \pm SEM. ${ }^{* * *} p<0.001,{ }^{* *} p<0.01$, ns not significant, compared to +ATR control, ANOVA with Holms-Sidak's post-hoc test. ATR: all-trans retinal. 

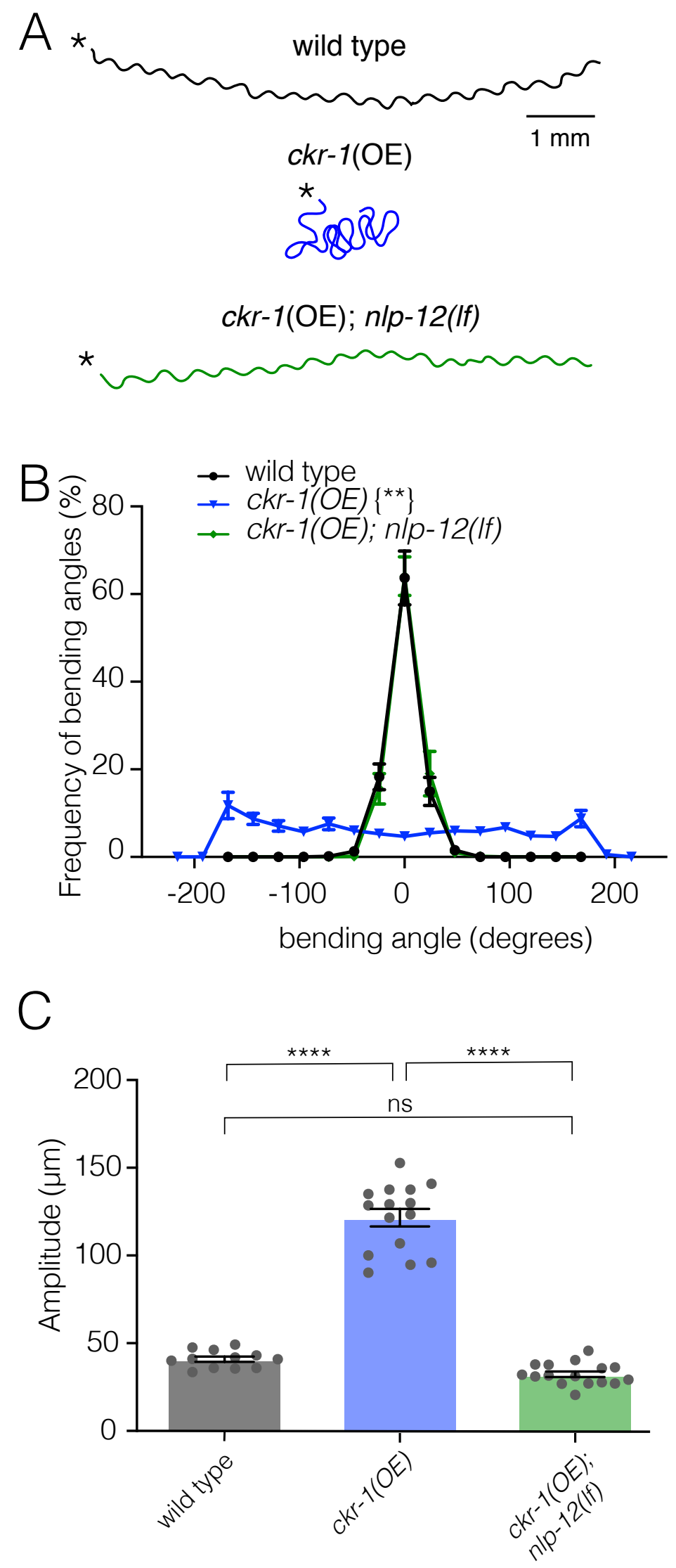
Figure 4. Elevated CKR-1 signaling enhances bending angle and amplitude in a $n / p-12$ dependent manner

(A) Representative movement trajectories of wild type (black), ckr-1(OE) (blue) and ckr-1(OE); nlp-12(If) (green) animals for 30 seconds on NGM agar plates seeded with OP50 bacteria. ckr$1(O E)$ refers to high copy expression of the wild type $c k r-1$ genomic locus (ufEx802). Note the increased frequency of high angle turns and convoluted track for $c k r-1(O E)$. These movement phenotypes are reversed by nlp-12 deletion. Scale bar, $1 \mathrm{~mm}$.

(B) Frequency distribution of body bending angles (mean \pm SEM) during forward runs (30 s) on plates thinly seeded with OP50 bacteria. Kolmogorov-Smirnov test: wild type vs ckr-1(OE) **, ckr-1(OE) vs ckr-1(OE); nlp-12(ok335) **, wild type vs ckr-1(OE); nlp-12(ok335) ns. ** p<0.01, ns not significant. wild type: $\mathrm{n}=8, c k r-1(O E): \mathrm{n}=10$, and $c k r-1(O E) ; n l p-12(I f): \mathrm{n}=10$.

(C) Comparison of the average body bend amplitude for the indicated genotypes. Bars represent mean \pm SEM. ${ }^{* * * *} p<0.0001$, ns not significant, ANOVA with Holms-Sidak's post-hoc test. wild type: $\mathrm{n}=12, c k r-1(O E): \mathrm{n}=15, c k r-1(O E) ; n l p-12(o k 335): \mathrm{n}=16$ 

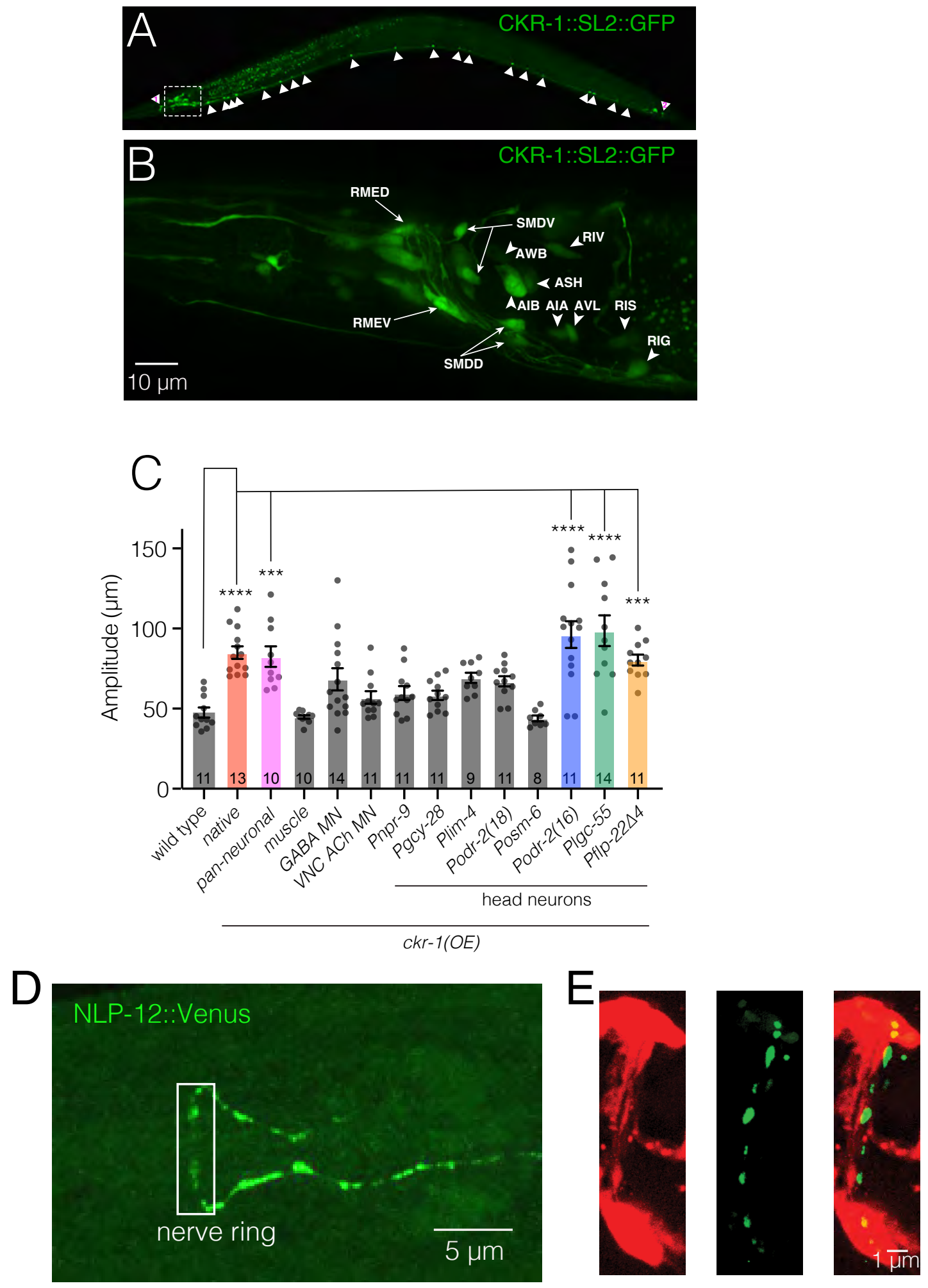

SMD::mCherry NLP-12::Venus

Merge 
Figure 5. ckr-1 functions in the SMD head motor neurons to modulate body bending (A) Confocal maximum intensity projection of adult expressing the Pckr-1::ckr-1::SL2::GFP reporter. Note expression in multiple head neurons (white box) and a subset of ventral nerve cord motor neurons (white arrowheads).

(B) Confocal maximum intensity projection of the head region of adult expressing the Pckr-1::ckr1::SL2::GFP reporter. Scale bar, $10 \mu \mathrm{m}$. See Fig. S3 and Table S2 for additional expression information.

(C) Quantification of average body bend amplitudes (mean \pm SEM) for ckr-1 overexpression in the indicated cell types. Promoters used for listed cell types: pan-neuronal Prgef-1, muscle Pmyo-3, GABA motor neurons Punc-47, cholinergic ventral cord motor neurons Punc-17 $\beta$. See Table S3 for details about cellular expression of promoters used for head neurons.

${ }^{* * * *} p<0.0001,{ }^{* * *} p<0.001$, ANOVA with Holms-Sidak's post-hoc test. Numbers within bars indicate $\mathrm{n}$ for each genotype.

(D) Confocal maximum intensity projection of the nerve ring region of a transgenic animal expressing Pnlp-12::NLP-12::Venus. Note the high levels of NLP-12::Venus in the nerve ring. White box indicates approximate nerve ring region where close localization of NLP-12 clusters to SMD processes has been shown in panel E. Scale bar, $5 \mu \mathrm{m}$.

(E) Confocal maximum intensity projection of the nerve ring region of a transgenic animal expressing Pnlp-12::NLP-12::Venus (DVA) and Pflp-22 $4:: m$ Cherry (SMD). Note the close localization of NLP-12::Venus dense core vesicle clusters to the SMD process. Scale bar, $1 \mu \mathrm{m}$. 


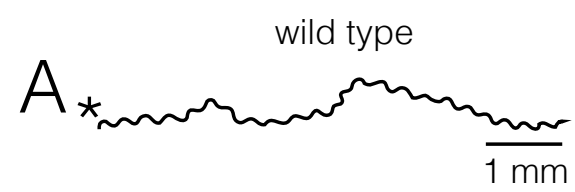

SMD::ckr-1(OE) [Podr-2(16)::ckr-1]

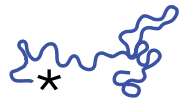

SMD::ckr-1(OE) [Pflp-22 $44:: c k r-1]$

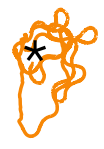

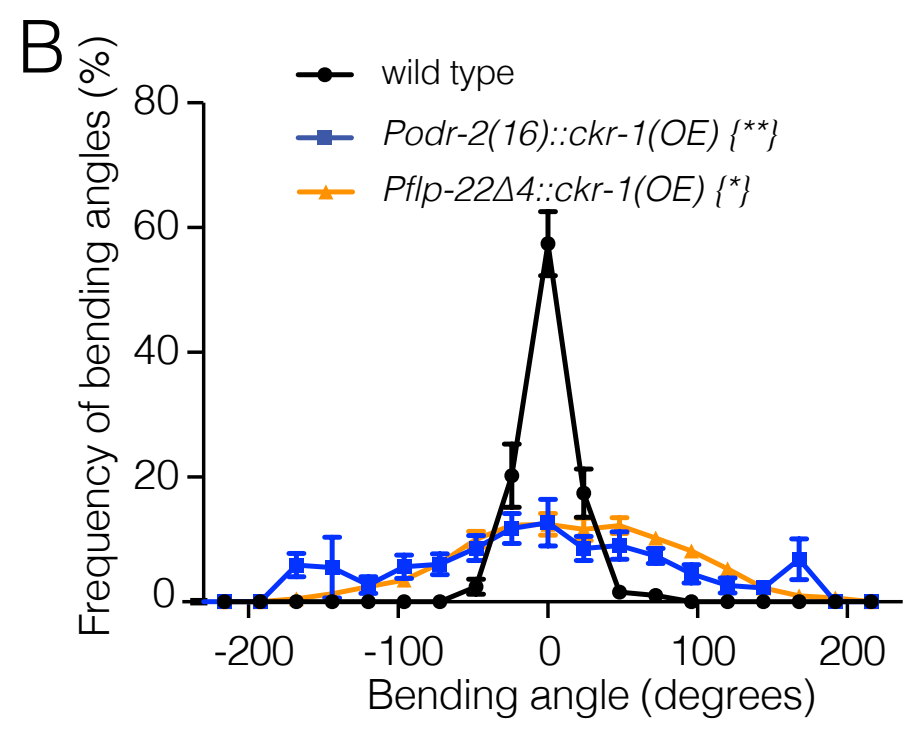

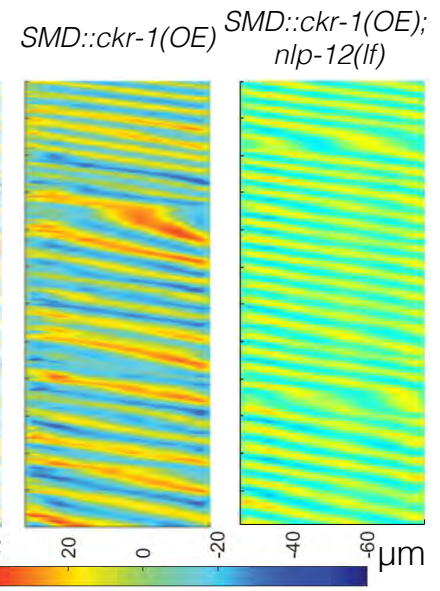

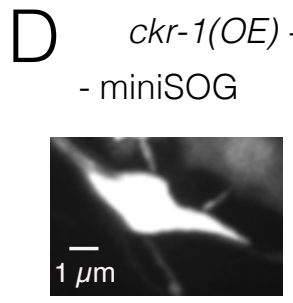

$\frac{* m}{1 m m} h$ $n / p-12(O E)$

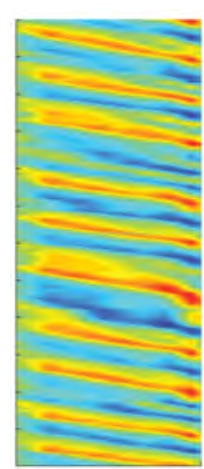

Head $\longrightarrow$ Tail
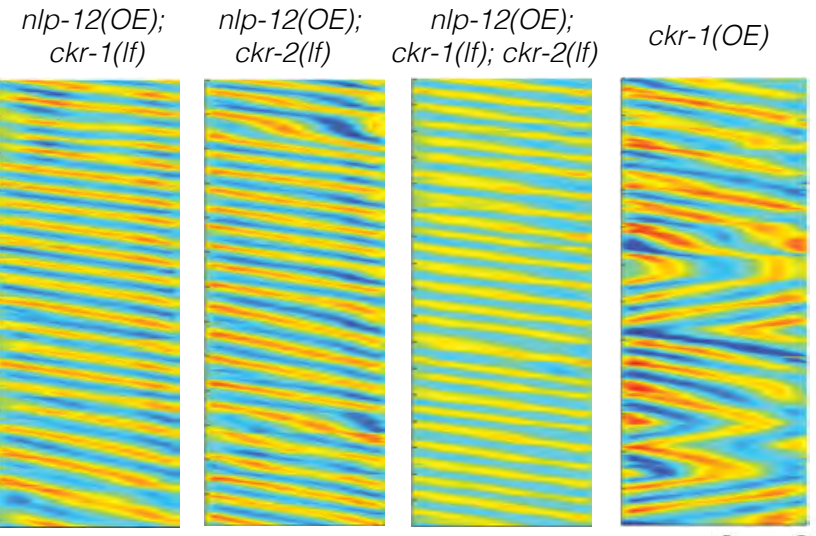

E

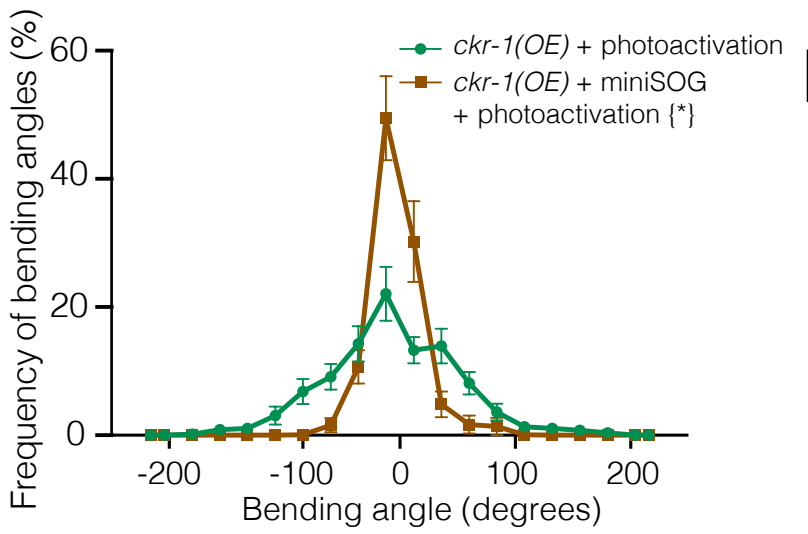

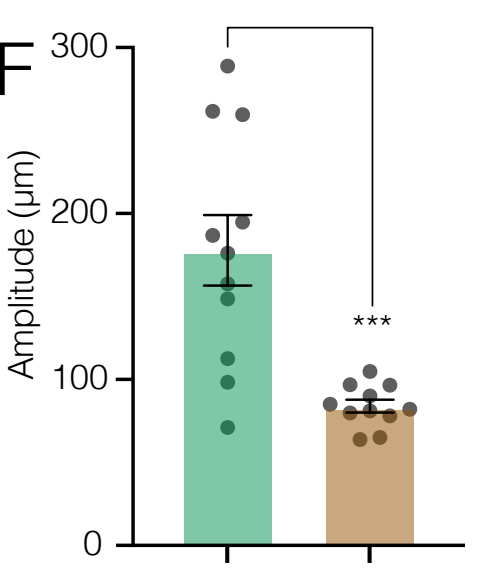

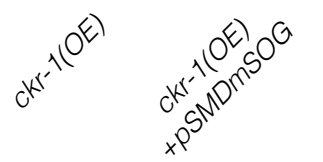


Figure 6. Ablation of SMD motor neurons abolishes the effects of $c k r-1$ overexpression

(A) Representative tracks (1 minute) for indicated genotypes. Asterisks indicate position of animal at the beginning of recordings. Note increased reorientations and body bending depth in the tracks with cell-specific $c k r-1$ overexpression. Scale bar, $1 \mathrm{~mm}$.

(B) Average body bending angle distribution (mean \pm SEM) for the indicated genotypes. High level expression of $c k r-1$ in SMDs using the odr-2(16) or flp-22 $\Delta 4$ promoters increases bending angle. Kolmogorov-Smirnov test: wild type vs Podr-2(16)::ckr-1(OE) **, wild type vs Pflp-22 4::ckr-1(OE) *, ** $p<0.01,{ }^{*} p<0.05$. wild type $\mathrm{n}=9$ (black circles), Podr-2(16)::ckr1(OE): $\mathrm{n}=8$ (blue squares), Pflp-22 $4:: c k r-1(O E): \mathrm{n}=11$ (orange triangles).

(C) Representative body curvature kymographs for worm locomotion during basal locomotion for indicated genotypes. Head to tail orientation along the horizontal axis in each kymograph is left to right as indicated for wild type. Time is indicated along the vertical axis from 0 to 1 minute.

(D) Top, representative fluorescent images of SMD motor neuron in ckr-1(OE) animals without (left) or with (right) miniSOG expression 16 hours following photoactivation. Bottom, representative $30 \mathrm{~s}$ track for control $c k r-1(O E)$ (-miniSOG, left) animal or SMD ablated $c k r-$ 1(OE) (+miniSOG, right) animal 16 hours after photostimulation. Scale bar, $1 \mu \mathrm{m}$.

(E) Average body bending angle distribution (mean \pm SEM) for control $c k r-1(O E)$ (green circles, $\mathrm{n}=11$ ) and SMD ablated $c k r-1(O E)$ (brown squares, $\mathrm{n}=11$ ) animals. SMD ablation reduces the frequency of large bending angles produced by $c k r-1(O E)$. Kolmogorov-Smirnov test: * $p<0.05$

(F) Comparison of average body bending amplitude for control ckr-1(OE) ( $\mathrm{n}=11)$ and SMD ablated $c k r-1(O E)(n=11)$. SMD ablation significantly reduces the enhanced body bending amplitude observed by $c k r-1(O E)$. Bars represent mean \pm SEM. ${ }^{* * *} p<0.001$, Student's t test. 
bioRxiv preprint doi: https://doi.org/10.1101/2020.04.27.064550; this version posted June 28, 2021. The copyright holder for this preprint (which was not certified by peer review) is the author/funder. All rights reserved. No reuse allowed without permission.
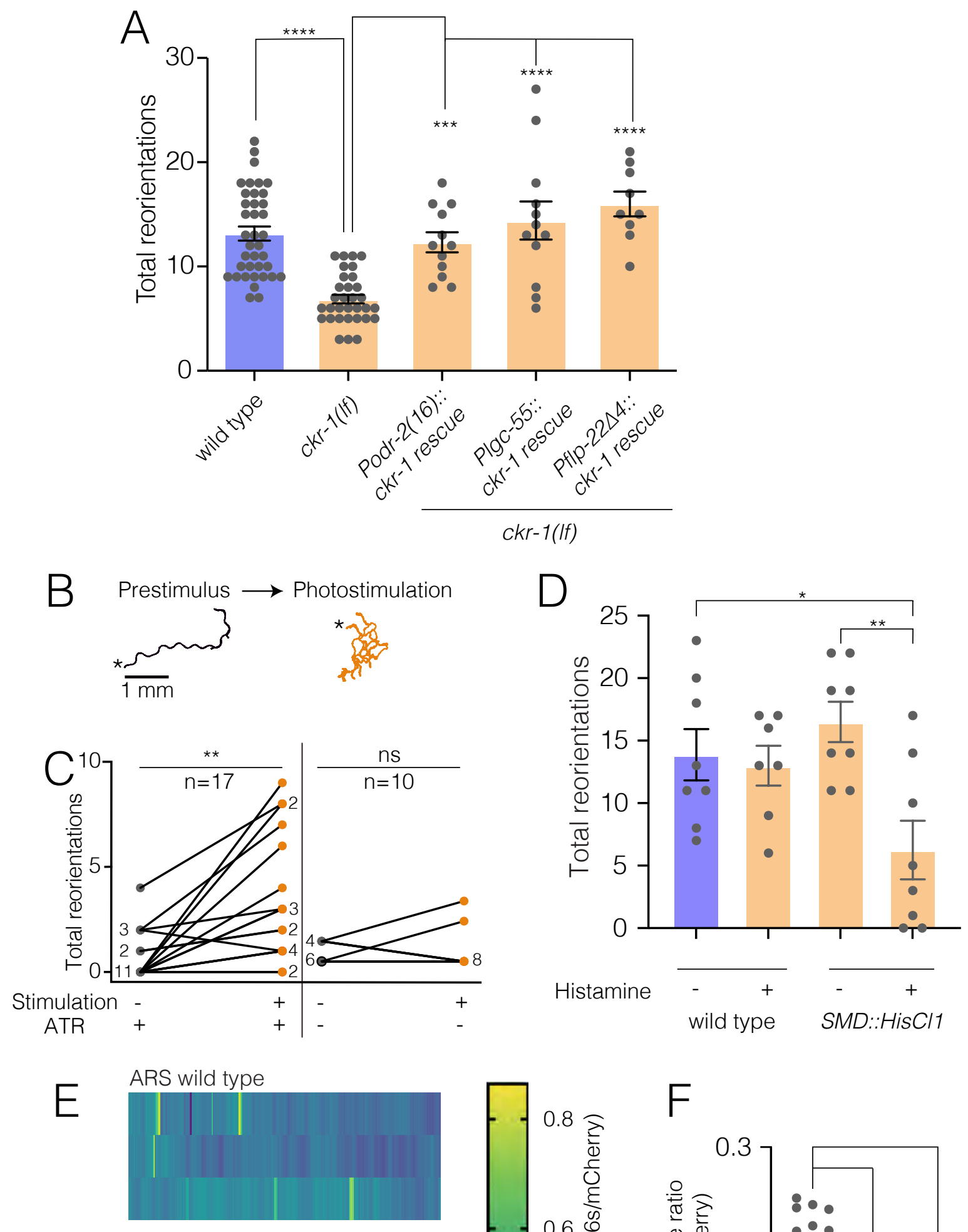

ARS ckr-1(If)

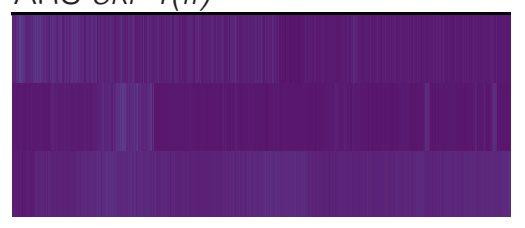

Dispersal wild type

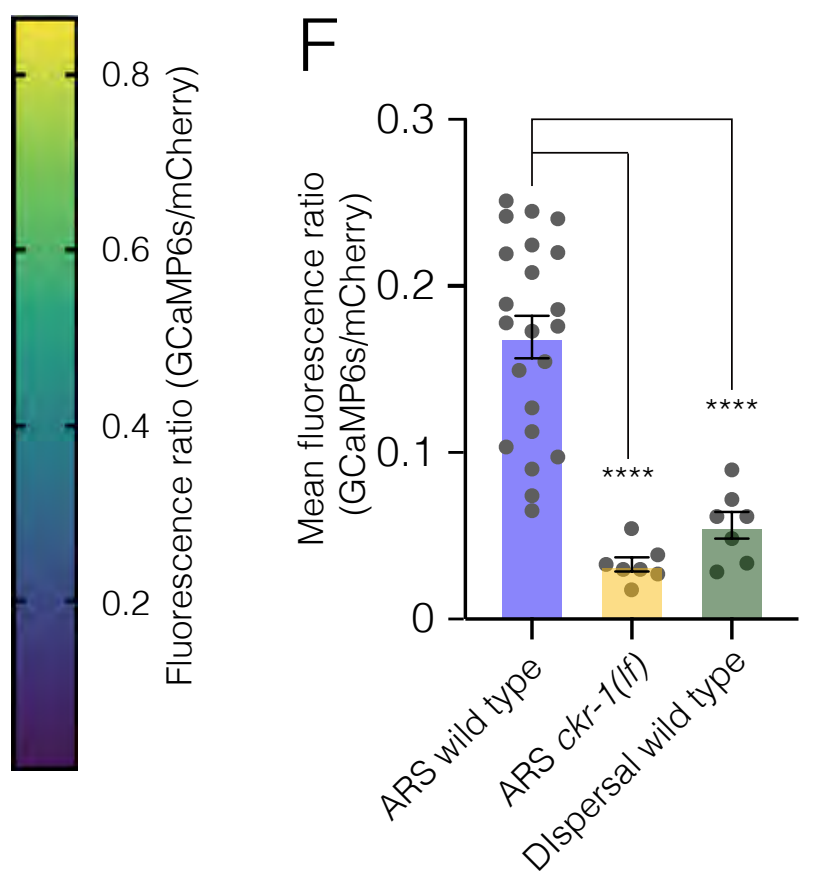




\section{Figure 7. NLP-12/CKR-1 excitation of the SMD neurons promotes reorientations}

(A) Total reorientations measured during 0-5 minutes following removal from food for the genotypes indicated. $c k r-1$ rescue refers to expression of wild type $c k r-1(5 \mathrm{ng} / \mu \mathrm{L})$ in $c k r-1(0 k 2502)$ animals using the indicated promoters. Bars represent mean \pm SEM. ${ }^{* * *} p<0.0001,{ }^{* * *} p<0.001$ ANOVA with Holms-Sidak's post-hoc test. wild type: $\mathrm{n}=38, c k r-1($ If): $\mathrm{n}=32, \operatorname{Podr}-2(16):: c k r-1$ rescue: $\mathrm{n}=12$, Plgc-55::ckr-1 rescue: $\mathrm{n}=12$, Pflp-22( $\Delta 4):: c k r-1$ rescue: $\mathrm{n}=9$.

(B) Representative tracks (1 minute) on thinly seeded NGM agar plates prior to (left) and during photostimulation (right) for transgenic animals expressing Podr-2(16)::Chrimson. Scale bar, $1 \mathrm{~mm}$. Asterisks $\left(^{*}\right)$ indicate position of worm at start of recording.

(C) Left, quantification of reorientations for individual animals over 1 minute durations prior to (prestimulus) and during photostimulation (+ATR). Right, quantification of reorientations for individual animals prior to and during photostimulation in control animals (-ATR). Black circles, reorientations during prestimulus. Orange circles, reorientations during photostimulation. Numbers adjacent to circles indicate number of overlapping data points. ${ }^{* *} p<0.01$, ns not significant. Paired t-test. ATR: all trans retinal.

(D) Quantification of reorientations for wild type and transgenic animals, where SMD activity was silenced (Pflp-22 4 4::His-Cl1::SL2::GFP), in the presence and absence of Histamine. ${ }^{* *} p<0.01$, * $p<0.05$, ANOVA with Holms-Sidak's post-hoc test. wild type: -Histamine $n=8$, +Histamine $n=7$, pSMD::HisC11::SL2::GFP: -Histamine $\mathrm{n}=8$, +Histamine: $\mathrm{n}=8$

(E) Representative heat maps showing activity in SMDs in transgenic animals (PfIp-22 4::GCaMP6s::SL2::mCherry) during ARS in wild type, dispersal in wild type, and ARS in $c k r-1$ (ok2502) background. Each row represents one animal over a duration of 1 minute. Note the higher activity in SMDs during ARS, which is significantly reduced in $c k r-1$ (If), and during dispersal.

(F) Quantification of mean fluorescence ratio (GCaMP6s/mCherry) per animal. ${ }^{* * * *} p<0.0001$, ANOVA with Holms-Sidak's post-hoc test. ARS wild type: $n=22$, ARS ckr-1(ok2502): $n=7$, Dispersal wild type: $n=7$. 
bioRxiv preprint doi: https://doi.org/10.1101/2020.04.27.064550; this version posted June 28, 2021. The copyright holder for this preprint (which was not certified by peer review) is the author/funder. All rights reserved. No reuse allowed without permission.

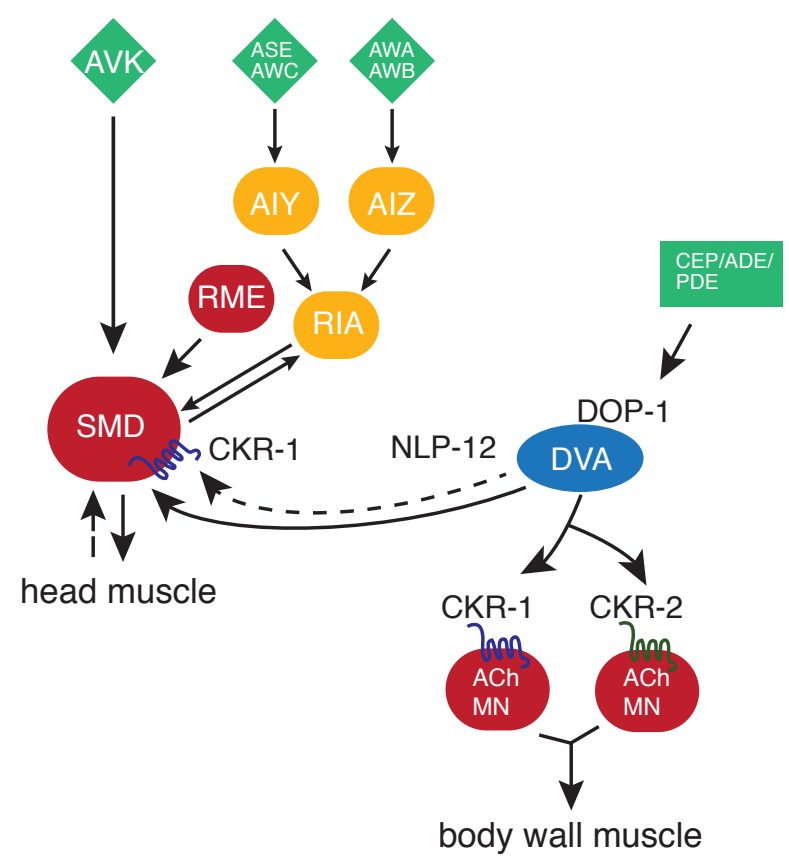




\section{Figure 8. Proposed model for NLP-12 action through CKR-1 and CKR-2}

During basal locomotion, NLP-12 activation of CKR-1 and CKR-2 GPCRs in ventral nerve cord motor neurons regulates body bending. During local searching, NLP-12 acts primarily through CKR-1 in SMD motor neurons to promote increased turning, trajectory changes and enhance body bending. Solid arrows indicate known synaptic connections, dotted arrows indicate extrasynaptic. Sensory neurons (green), head interneurons (orange), and motor neurons (red). Olfactory sensory neurons: AWA, AWB, AWC, ASE. 
bioRxiv preprint doi: https://doi.org/10.1101/2020.04.27.064550; this version posted June 28, 2021. The copyright holder for this preprint (which was not certified by peer review) is the author/funder. All rights reserved. No reuse allowed without permission.

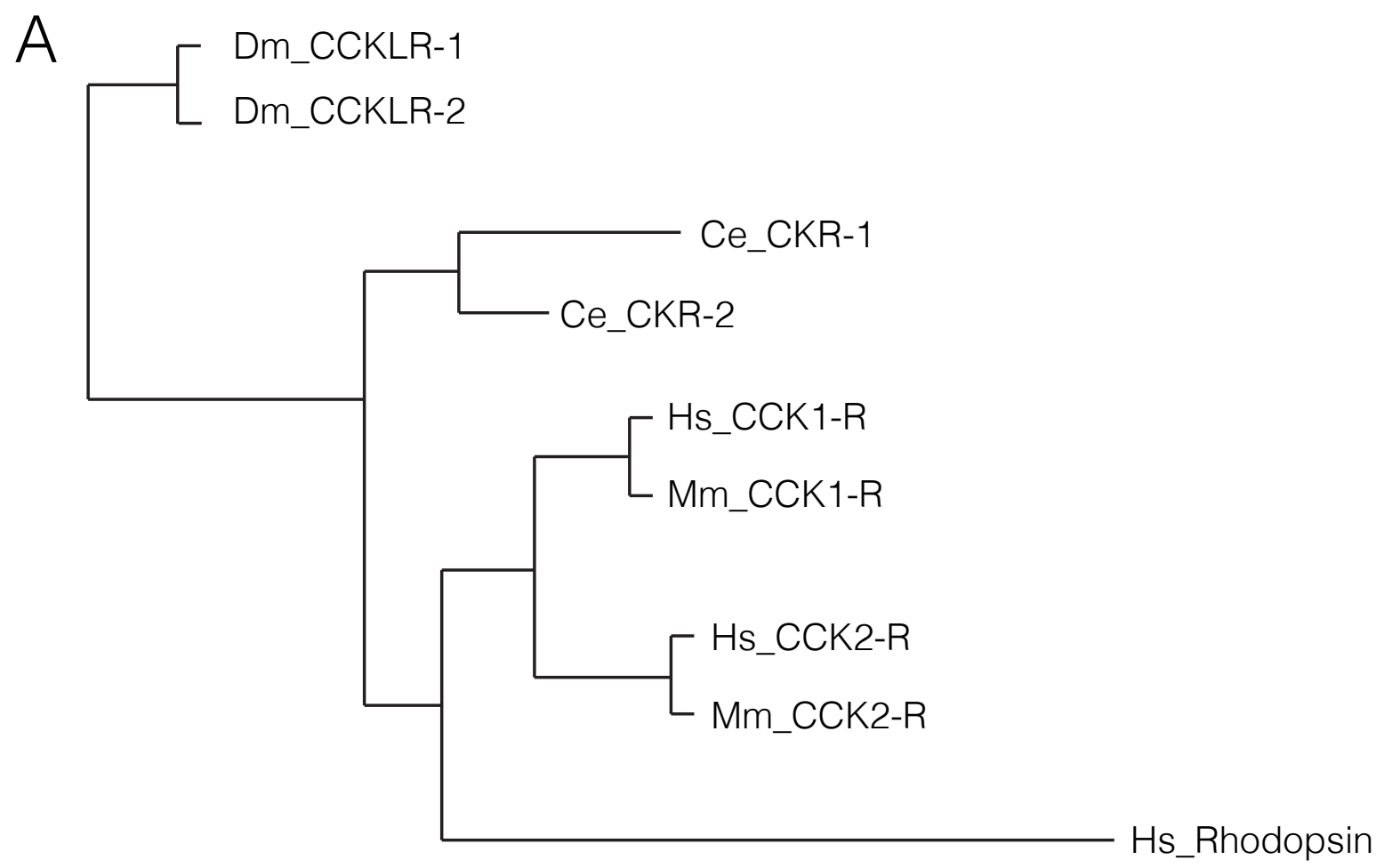

Ce CKR-1 Hu CCK1-R Hu CCK2-R

1
1
1
1
1

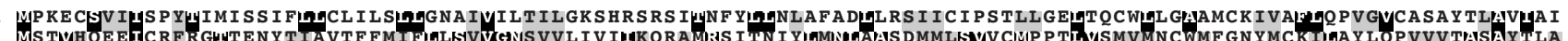

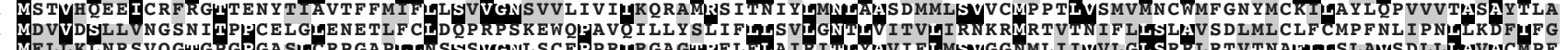

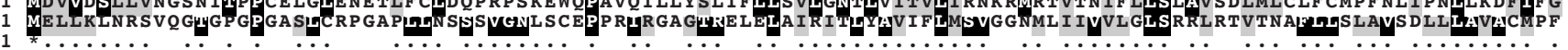

Ce CKR-1 Hu CCK1-R Hu CCK2-R

111 ERYYA I CR PLE SRKWQTLKRALI TISLVWCF S FSANLT SLF LYDHN PGKFHC DSHK G PLVDF I Y QLYL TF TLLFV PLALMVGL Y GNV I I TLINT ALNSDHFHVE OOM I E KT

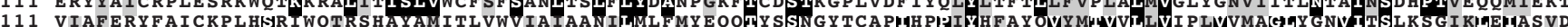

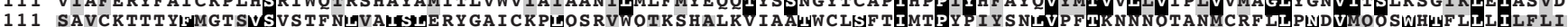
111 TLLPNLMGTF IFG TVICKAVSYLMGVSVS STLSLVAIAEERYSAICRPLQARVWOTRSHAARVIVATWLLSGLLMVYPVY TVVOPVGPRVLOCVHRWFSARVROTWS

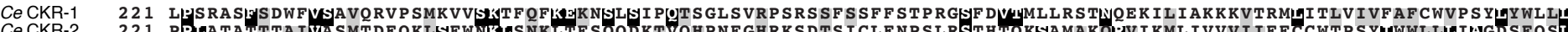
Ce CKR-2 Hu CCK1-R Hu CCK2-R

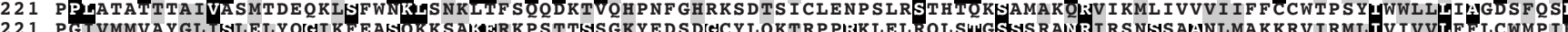

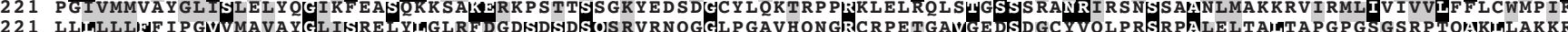
221 LLLLLEF I PGVVMAVAYGL ISRE LYLGLRFDGDSDSDSOSRVRNQGGLPGAVHQNGRCRPETGAVGEDSDGCYVQLPRSRPALELTALTAPGPGSGSRPTQALELAKKR

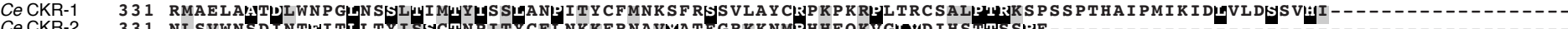

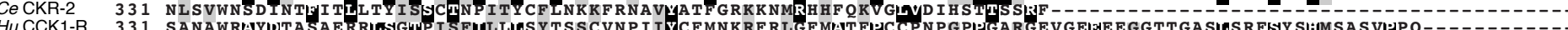

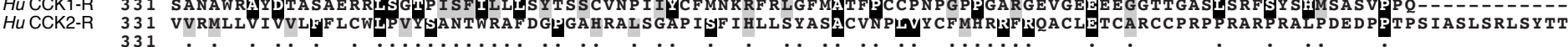
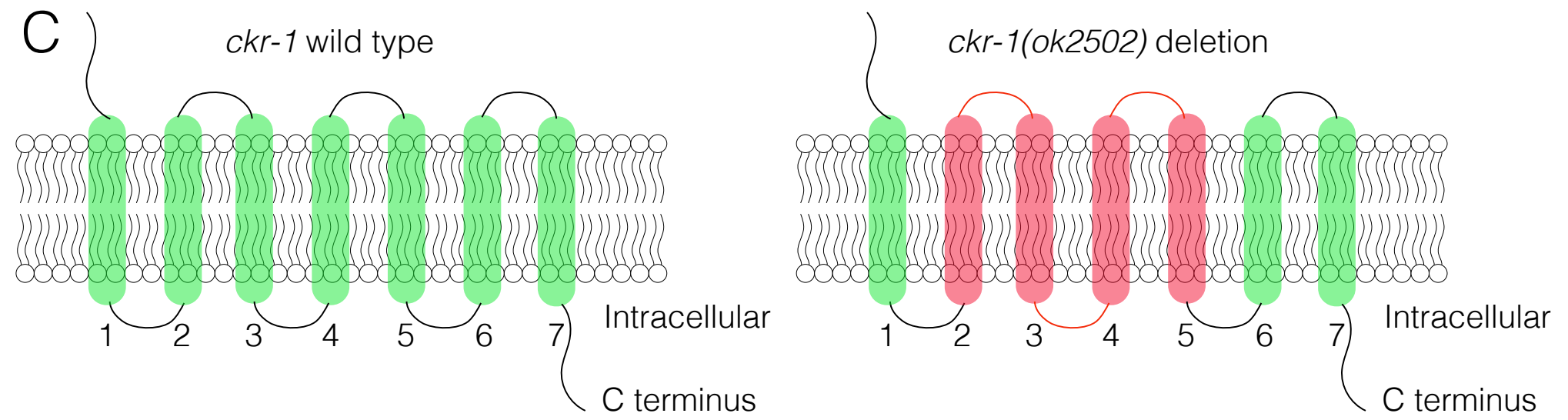


\section{Figure S1}

(A) Dendrogram (generated using Phylogeny, $\mathrm{fr}^{64}$ ) showing the predicted relationship between

Drosophila (Dm_CCKLR-1/2), C. elegans (Ce_CKR-1/2), mouse (Mm) and human (Hs) CCK1/2-R GPCRs.

(B) Boxshade alignment of C. elegans CKR-1 and CKR-2 with Human CCK-1 and CCK-2

receptors. Black shading indicates identical amino acids, while grey shading indicates similar amino acids. Red bar indicates the amino acids removed by ckr-1(ok2502) deletion.

(C) Schematic representation of CKR-1 GPCR membrane topology and domains affected by the $c k r-1$ (ok2502) deletion (red shading). 


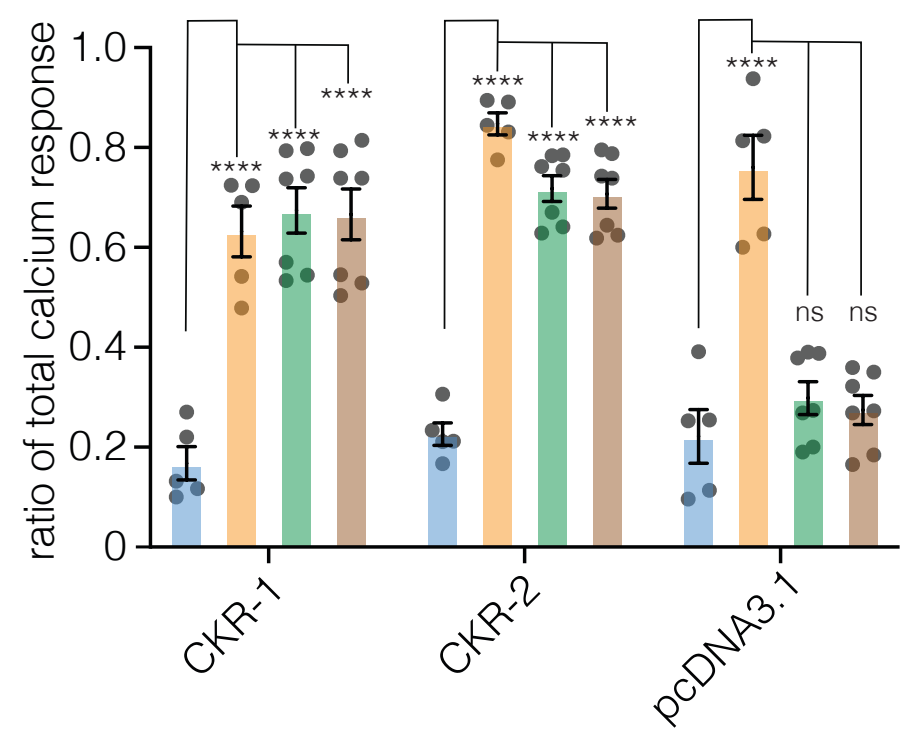

BSA

ATP

- NLP-12-1

NLP-12-2 
bioRxiv preprint doi: https://doi.org/10.1101/2020.04.27.064550; this version posted June 28, 2021. The copyright holder for this preprint (which was not certified by peer review) is the author/funder. All rights reserved. No reuse allowed without permission.

\section{Figure $\mathbf{S 2}$}

NLP-12 peptides activate CKR-1 and CKR-2 in vitro. NLP-12-1 and NLP-12-2 elicit Ca ${ }^{2+}$ responses in cells expressing CKR-1 or CKR-2, but not in cells transfected with an empty pcDNA3.1 vector. Bar graphs indicate the ratio of total $\mathrm{Ca}^{2+}$ response of $\mathrm{CHO}$ cells expressing $\mathrm{CKR}-1, \mathrm{CKR}-2$ or pcDNA3.1 empty vector, challenged with $10 \mu \mathrm{M}$ of NLP-12 peptides $(n=7)$, BSA (negative control, $n=5$ ) or ATP (positive control, $\mathrm{n}=5$ ). Ratio of total $\mathrm{Ca}^{2+}$ response is calculated as peptide-evoked response normalized to the total $\mathrm{Ca}^{2+}$ response. Data were analyzed by two-way ANOVA; ${ }^{* * *} \mathrm{p}<0.0001$; ns, not significant $(p>0.05)$. 


\section{A Forward reorientation}
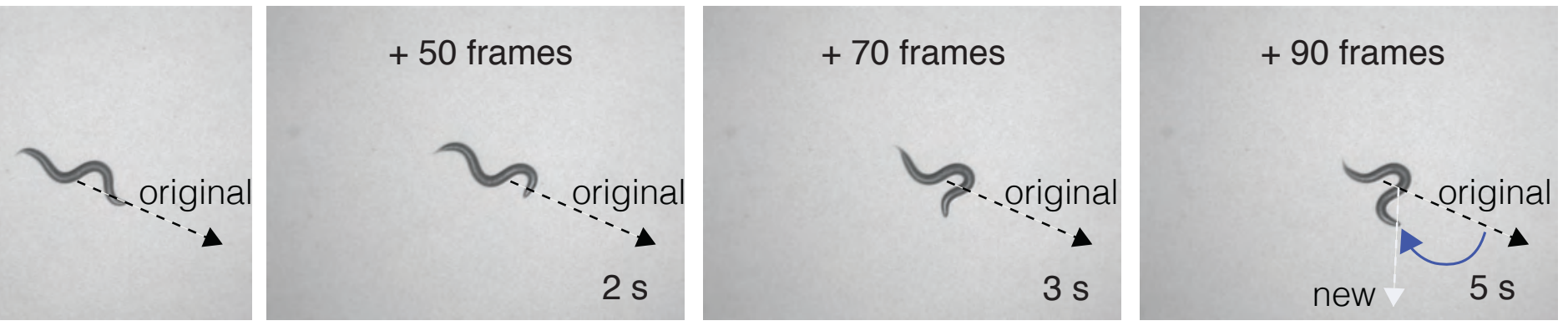

\section{B Reversal-coupled omega turn reorientation}
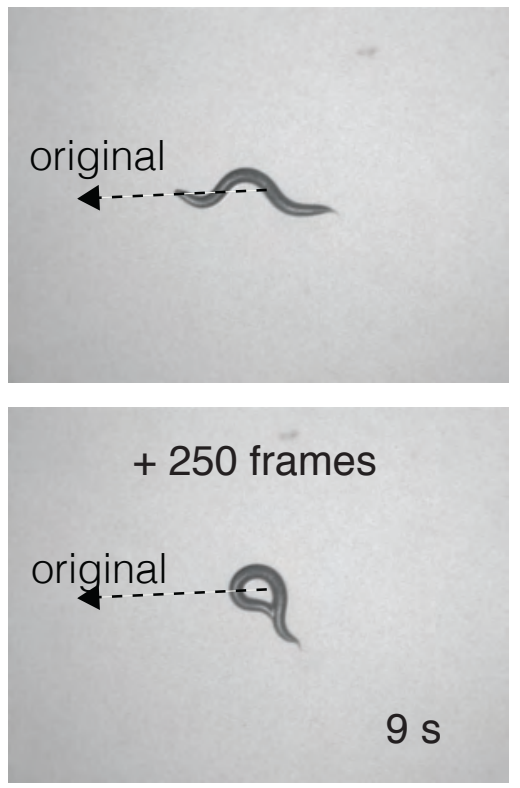
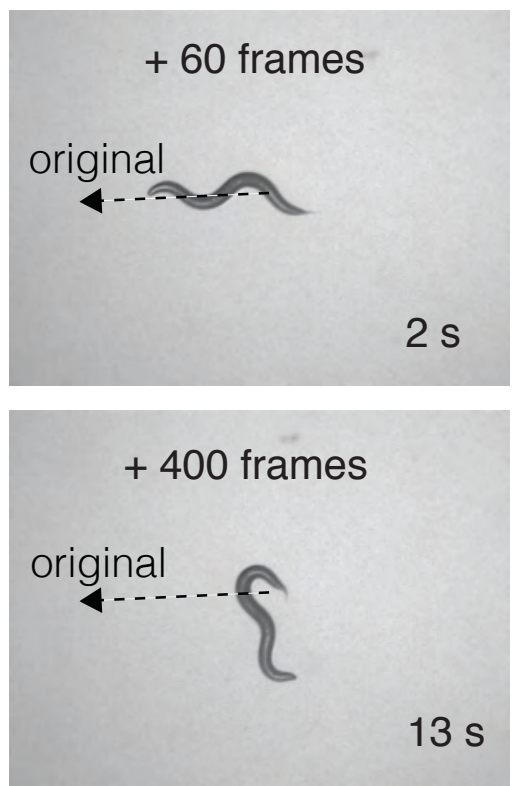
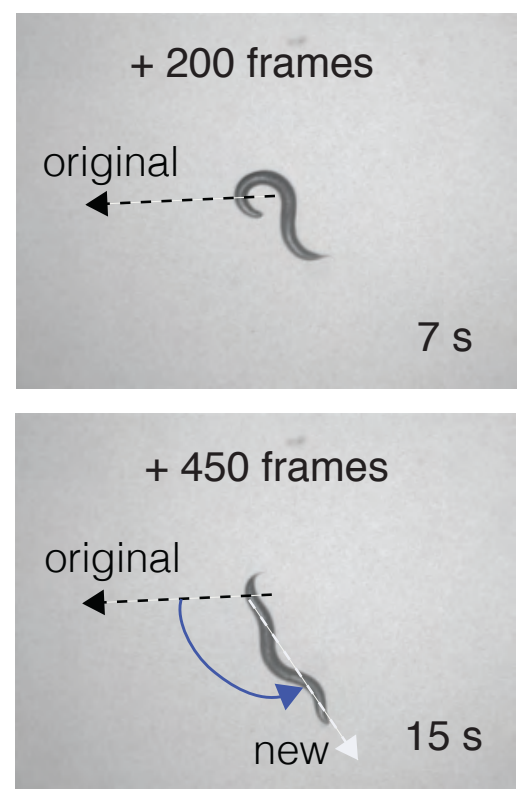
bioRxiv preprint doi: https://doi.org/10.1101/2020.04.27.064550; this version posted June 28, 2021. The copyright holder for this preprint (which was not certified by peer review) is the author/funder. All rights reserved. No reuse allowed without permission.

\section{Figure S3}

Sequential snapshots of frames from a representative reorientation, for forward reorientations (A) and reversal-coupled omega turn mediated reorientations (B). Frame \#s and time points are indicated in each panel. Frame numbers and time points indicated are relative to first image in each sequence, which represents the start point (frame 0 , time $0 \mathrm{~s}$ ) when the reorientation event began, and the last frame was when the reorientation was completed. Black dashed line shows the original trajectory, and white dashed line the new trajectory upon completion of the reorientation. Blue angle shows the measured change in trajectory (degrees). 

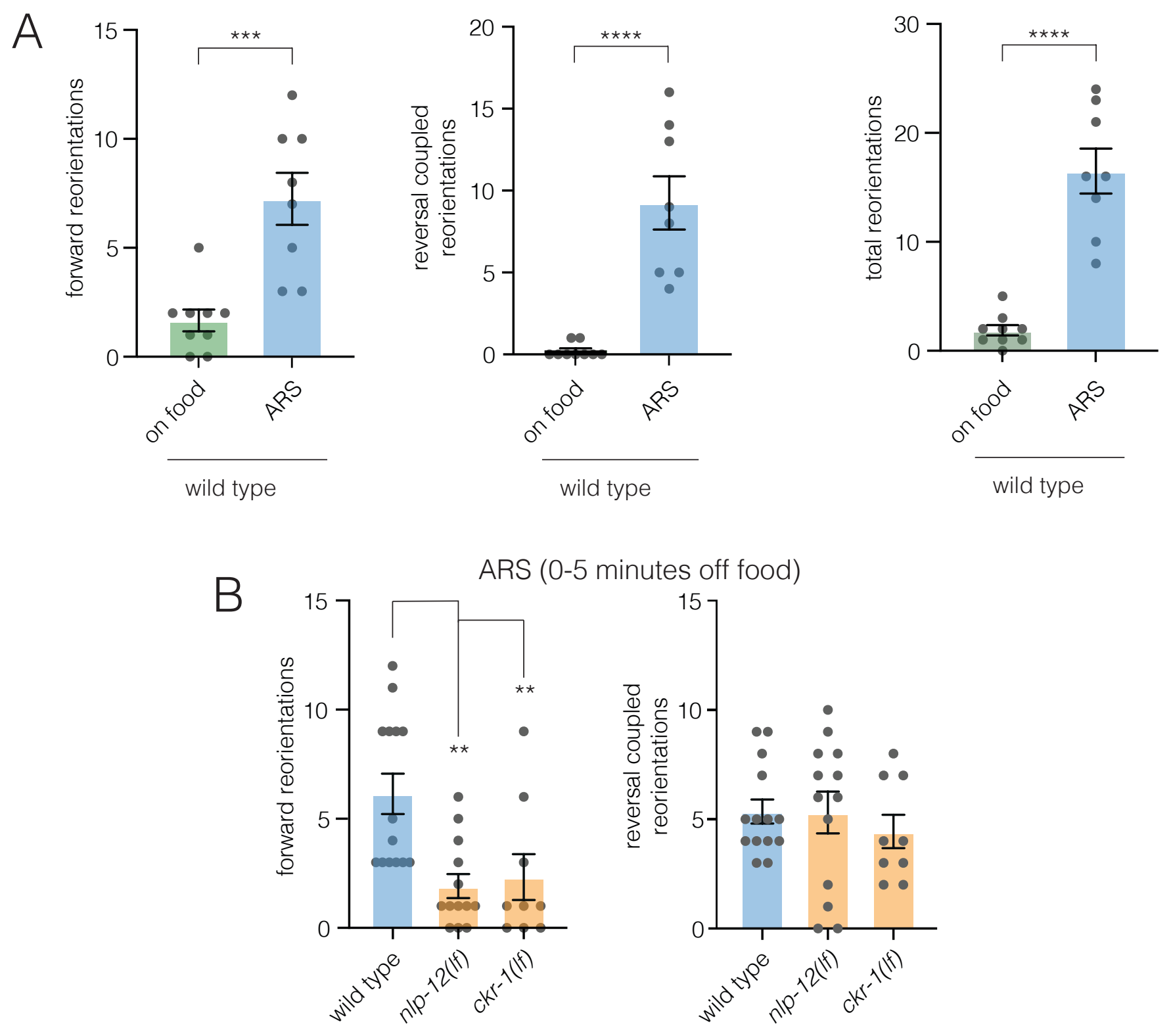


\section{Figure $\$ 4$}

(A) Quantification of reorientations during ARS (0-5 minutes following removal from food) compared to animals on food. Note the increased number of forward and reversal coupled reorientations. Bars represent mean \pm SEM. ${ }^{* * *} p<0.0001,{ }^{* * *} p<0.001$, Student's t test. wild type on food: $n=9$, wild type ARS: $n=8$

(B) Quantification of reorientations during ARS (0-5 minutes following removal from food) for the genotypes indicated. Note the number of forward reorientations during ARS are significantly decreased in nlp-12(ok335) and ckr-1(ok2502) animals. However, reversal coupled reorientations are unaffected. Bars represent mean \pm SEM. ${ }^{* *} p<0.01$, ANOVA with HolmsSidak's post-hoc test. wild type: $n=14, n / p-129$ (ok335): n=13, ckr-1(ok2502): n=9. 

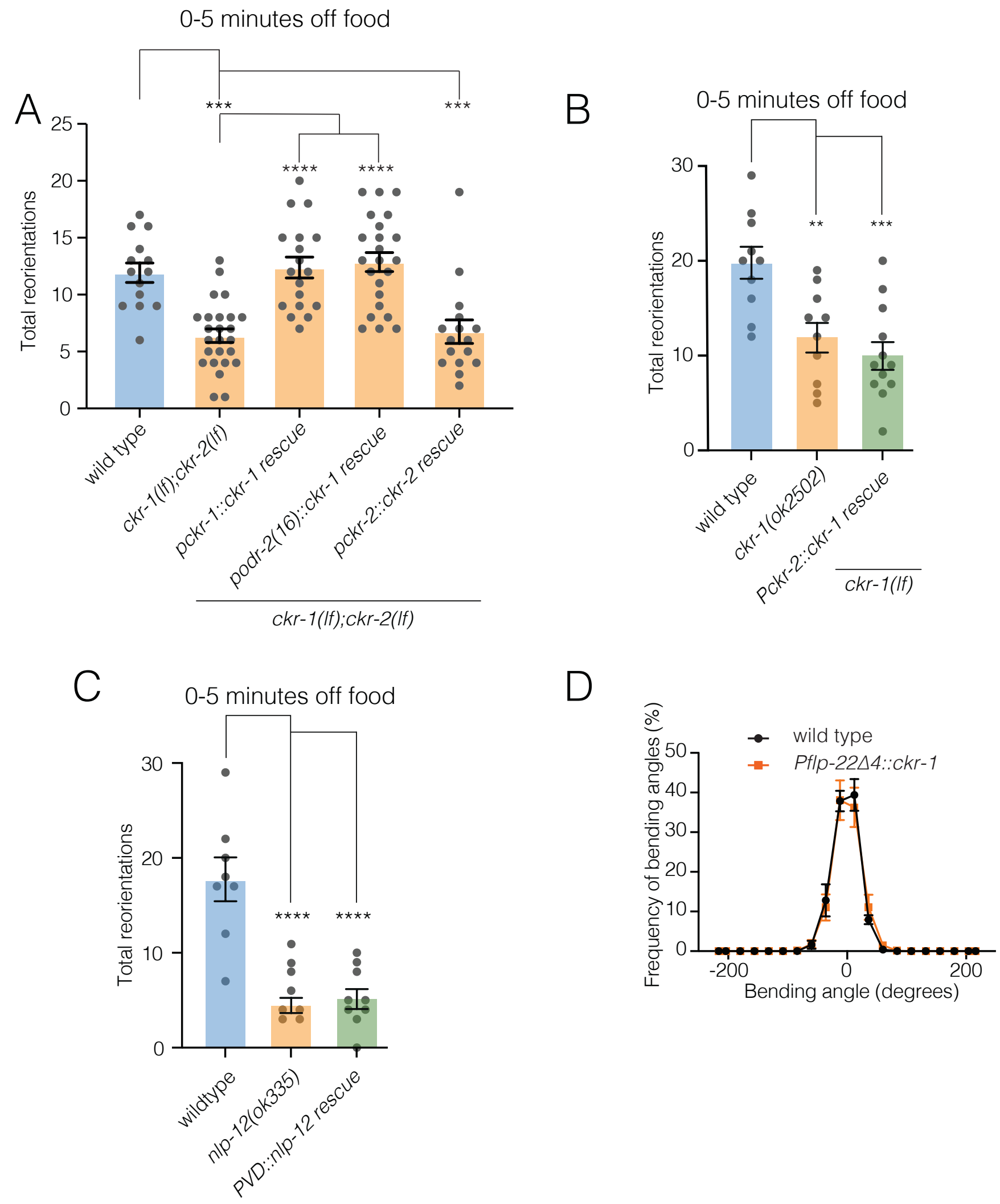


\section{Figure S5}

(A) Quantification of reorientations during ARS (0-5 minutes following removal from food) for the genotypes indicated. Rescue refers to transgenic expression of wild type $c k r-1$ or $c k r-2$ in ckr-1(ok2502);ckr-2(tm3082) mutants. Bars represent mean $\pm \mathrm{SEM} .{ }^{* * * *} \mathrm{p}<0.0001,{ }^{* * *}$ $p<0.001$, ANOVA with Holms-Sidak's post-hoc test. wild type: $n=14, c k r-1$ (ok2502);ckr2(tm3082): $\mathrm{n}=25$, Pckr-1::ckr-1 rescue: $\mathrm{n}=18$, Podr-2(16)::ckr-1 rescue: $\mathrm{n}=23$, Pckr-2::ckr-2 rescue: $n=16$.

(B) Quantification of reorientations during 0-5 minutes following removal from food for the genotypes indicated. Note expression of $c k r-1$ under the $c k r-2$ promoter does not rescue reorientations during ARS in ckr-1(ok2502) animals. Bars represent mean \pm SEM.

${ }^{* * *} p<0.001,{ }^{* *} p<0.01$, ANOVA with Holms-Sidak's post-hoc test. wild type: $n=10, c k r-$ 1(ok2502): $\mathrm{n}=10$, Pckr-2::ckr-1 rescue: $\mathrm{n}=12$.

(C) Quantification of reorientations during 0-5 minutes following removal from food for the genotypes indicated. Note expression of $n / p-12$ under the PVD specific promoter (ser-2prom3) does not rescue reorientations during ARS in nlp-12(ok335) animals. Bars represent mean \pm SEM. ${ }^{* * *} \mathrm{p}<0.0001$, ANOVA with Holms-Sidak's post-hoc test. wild type: $\mathrm{n}=8, n / p-12$ (ok335): $\mathrm{n}=8$, Pser-2prom3::nlp-12 rescue: $\mathrm{n}=9$.

(D) Average body bending angle distribution (mean \pm SEM) plotted for wild type control animals (solid black circles, $\mathrm{n}=8$ ) and Pflp-22 $4:: c k r-1$ (solid orange squares, $\mathrm{n}=8$ ). Low level (5 $\mathrm{ng} / \mu \mathrm{L})$ cell-specific expression of $c k r-1$ in SMDs in wild type did not alter body bending. Kolmogorov-Smirnov test not significant. 

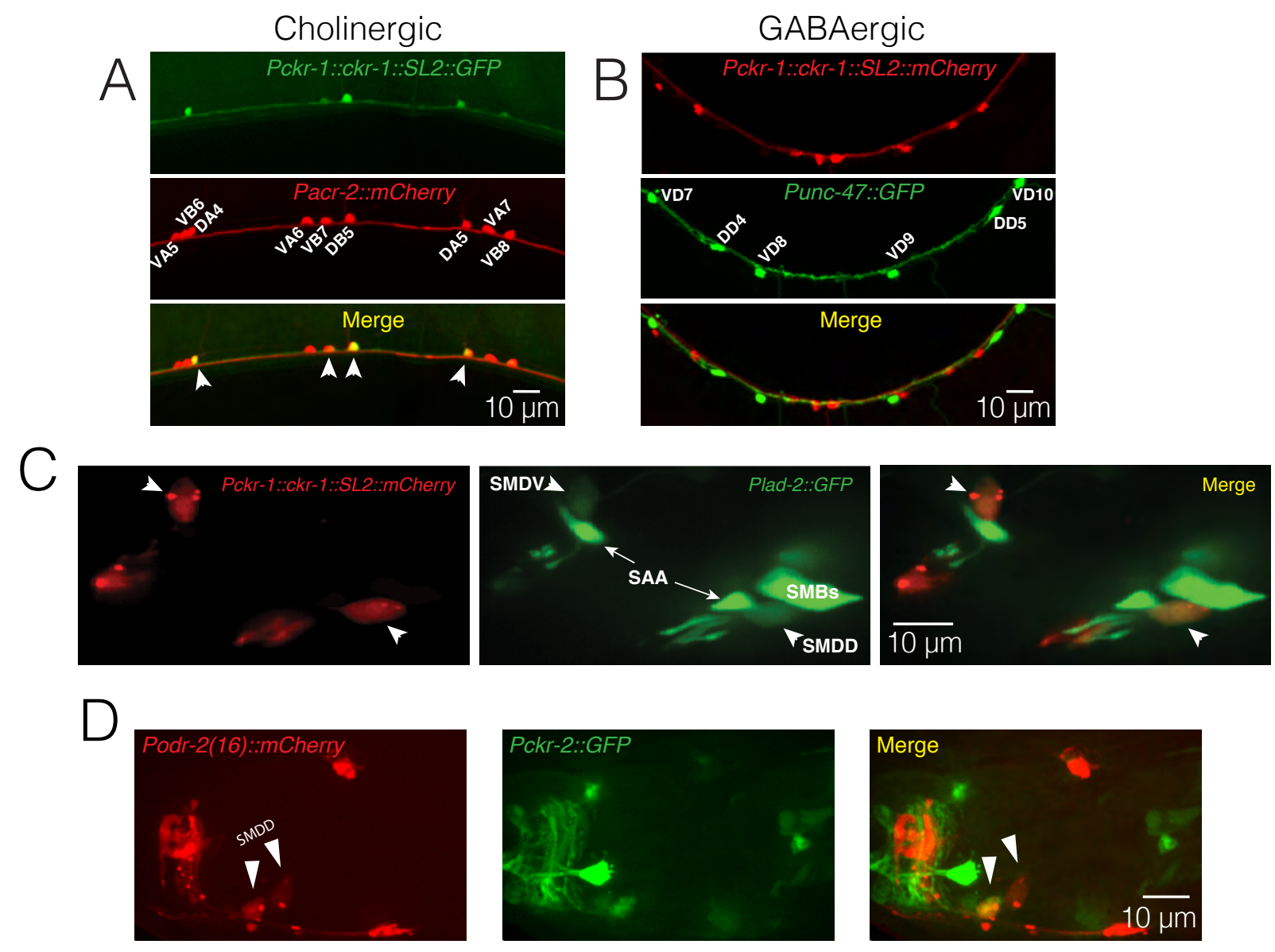


\section{Figure $\mathbf{S 6}$}

(A) Confocal maximum intensity projections of a segment of the ventral nerve cord of a transgenic animal coexpressing Pckr-1::ckr-1::SL2::GFP and the cholinergic reporter Pacr-2::mCherry. ckr-1 is expressed in the DA and DB motor neurons in the ventral nerve cord. Anterior is to the left in all panels. Scale bar, $10 \mu \mathrm{m}$.

(B) Confocal maximum intensity projections of a segment of the ventral nerve cord of a transgenic animal coexpressing Pckr-1::ckr-1::SL2::mCherry and the GABAergic reporter Punc-47::GFP.

(C) Confocal maximum intensity projections of optical sections with SMD fluorescence (GFP) from the head region of a transgenic animal expressing $c k r-1:: S L 2:: m$ Cherry (left panel) together with Plad2::GFP (middle panel). White arrowheads denote the SMD cell bodies in all cases. Note the colocalization of the red and green fluorescence exclusively in the SMD neurons (merge right panel).

(D) Confocal maximum intensity projections of optical sections with SMD fluorescence (mCherry) from the head region of a transgenic animal coexpressing Podr-2(16)::mCherry (left panel), and Pckr2::GFP (middle panel). Note weak ckr-2 expression in a single SMDD neuron (merge, right panel). 

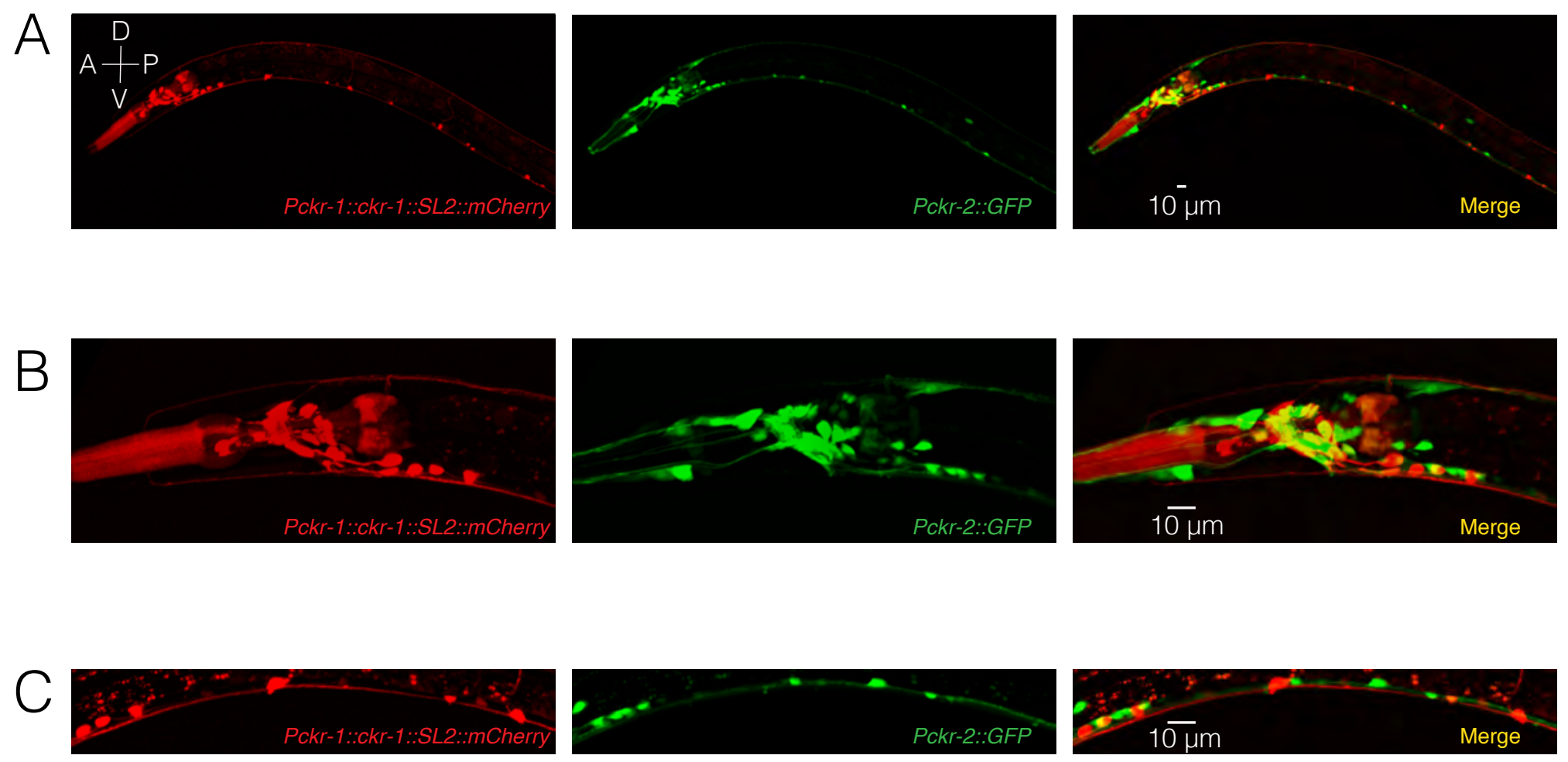


\section{Figure S7}

Confocal maximum intensity projections of transgenic worm expressing Pckr-1::ckr-1::SL2::mCherry and Pckr-2::GFP. (A) $c k r-1$ and $c k r-2$ expression in the entire worm. Both $c k r-1$ and $c k r-2$ are highly expressed in head neurons and ventral nerve cord motor neurons. However, there is very little overlap between expression of $c k r-1$ and $c k r-2$. (B) Magnified view of $c k r-1$ and $c k r-2$ expression in the head region. (C) Magnified view of $c k r-1$ and $c k r-2$ expression in the ventral nerve cord. Scale bar, $10 \mu \mathrm{m}$. 
A SMD::CKK-1(OE) [PHID-2244:CKK-1] B

SMD::ckr-1(OE); nlp-12(If)

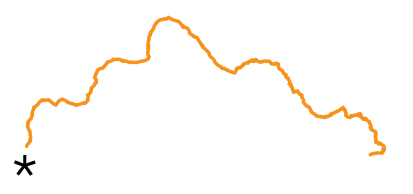

$\overline{1 \mathrm{~mm}}$

C

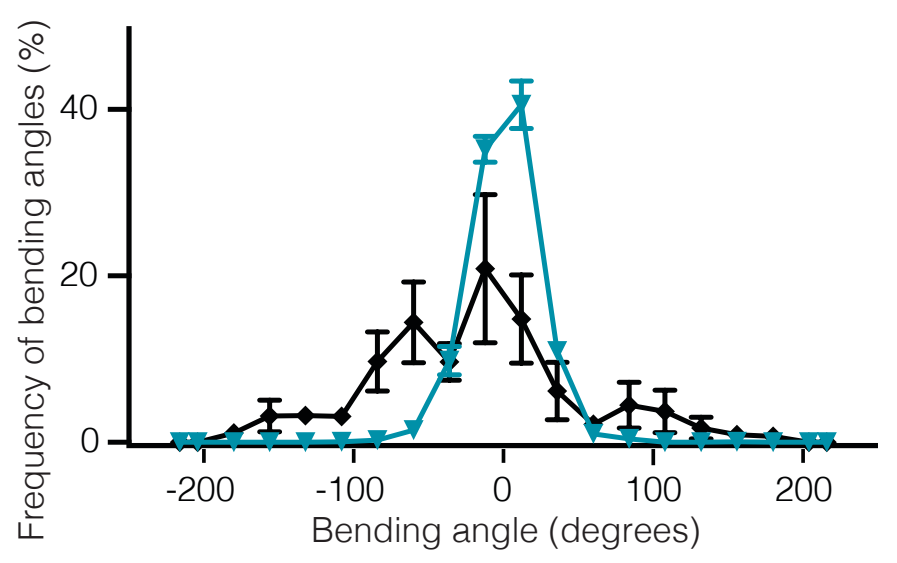

$\rightarrow$ wild type

$\neg \quad S M D:: c k r-1(O E)[P f l p-22 \Delta 4:: c k r-1]+S M D:: m i n i S O G$ [Pflp-22 4::miniSOG] (no photoactivation)

SMD::GFP [PfIp-22 $\triangle 4:: G F P]$

- Photoactivation

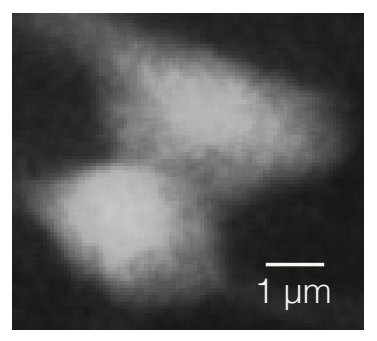

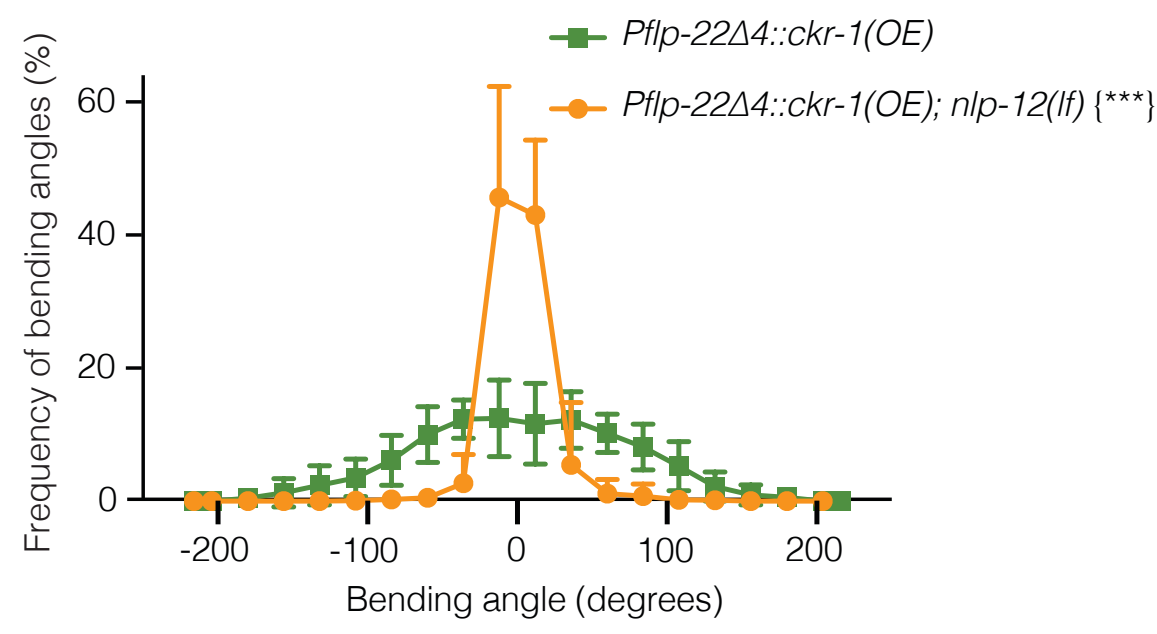

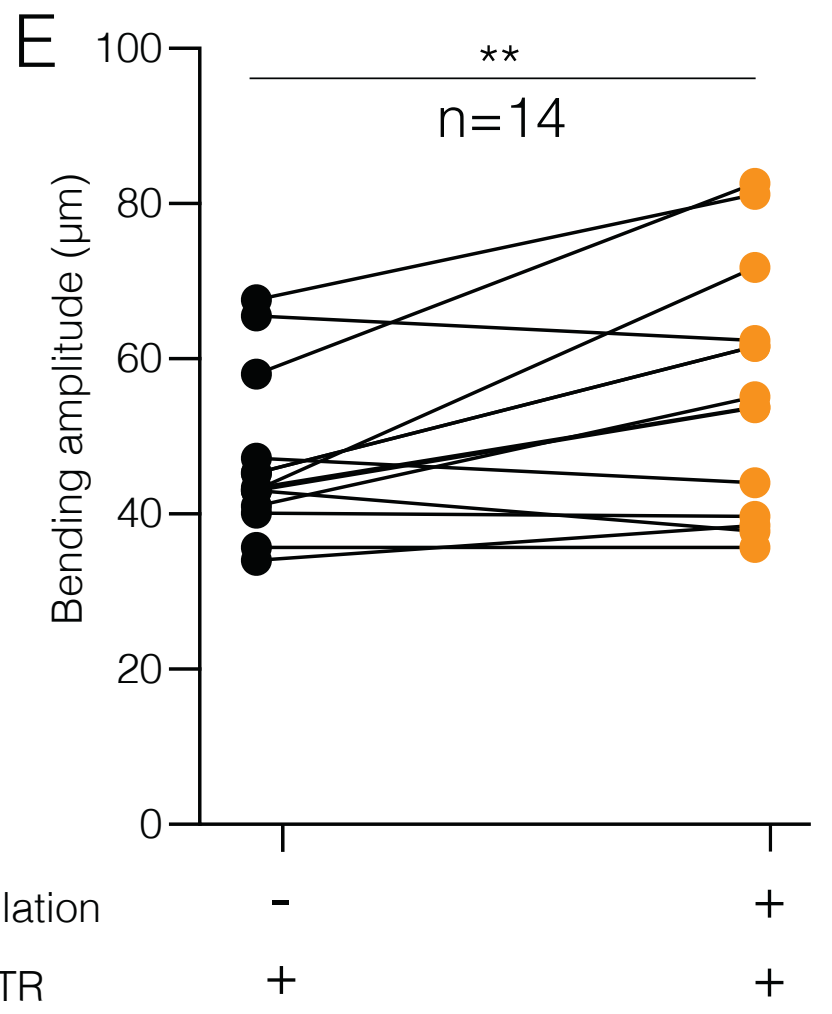




\section{Figure S8}

(A) Representative tracks (30 s) for transgenic animals with high levels of cell-specific ckr-1 overexpression (Pflp-22_4::ckr-1) in wild type (top) or $n / p-12$ deletion background (bottom). Asterisks indicate position of animals at the beginning of recording. Kolmogorov-Smirnov test *** $\mathrm{p}<0.001$. Scale bar, $1 \mathrm{~mm}$.

(B) Average bending angle distribution (mean \pm SEM) for SMD-specific ckr-1(OE) in wild type (green squares, $\mathrm{n}=11$ ) or $n l p-12$ (If) background (orange circles, $\mathrm{n}=10$ ).

(C) Average body bending angle distribution (mean \pm SEM) for $p S M D:: c k r-1(O E)$ animals expressing miniSOG in SMDs (Pflp-22 44::miniSOG), but not subjected to photoactivation (control, black diamonds) compared to wild type (blue triangles). $n=8$ for each group.

(D) Single confocal slices of GFP-labeled SMD neurons, following photoactivation (right) compared to control (-photoactivation, left), in transgenic animals without miniSOG expression. Photoactivation protocol does not alter SMD neuron morphology in the absence of miniSOG expression. Scale bar, $1 \mu \mathrm{m}$.

(E) Photostimulation of SMDs modestly increases body bending amplitude. ${ }^{* *} p<0.01$, paired Student's t-test. Black circles, reorientations during prestimulus. Orange circles, reorientations during photostimulation. 
bioRxiv preprint doi: https://doi.org/10.1101/2020.04.27.064550; this version posted June 28, 2021. The copyright holder for this preprint (which was not certified by peer review) is the author/funder. All rights reserved. No reuse allowed without permission.

\section{Table S1}

\section{Strains generated/used in this work}

\begin{tabular}{|c|c|c|}
\hline Strain & Genotype & Strain information \\
\hline IZ908 & $n / p-12(0 k 335) I$ & outcrossed from RB607 (CGC) \\
\hline IZ2287 & $c k r-1(0 k 2502) I$ & outcrossed from RB1923 (CGC) \\
\hline LSC0032 & ckr-2(tm3082) III & outcrossed from Japanese knockout allele \\
\hline IZ2304 & $c k r-1(0 k 2502)$ l; ckr-2(tm3082) III & \\
\hline IZ1152 & ufls104 & $\begin{array}{c}\text { Pnlp-12::nlp-12::nlp-12 3'UTR }(100 \mathrm{ng} / \mu \mathrm{L}) \text {, } \\
\text { Plgc-11::GFP [pHP6 (50 ng/ } \mu \mathrm{L})] \\
1.76 \mathrm{~kb} \text { PCR product containing the } n / p-12 \\
\text { promoter and genomic locus }(-354 \mathrm{bp} \text { to } \\
+1407 \text { bp relative to the transcriptional start). }\end{array}$ \\
\hline IZ1284 & ckr-1(ok2502) l; ufls104 & \\
\hline IZ1231 & $c k r-2(\operatorname{tm} 3082)$ III; ufls104 & \\
\hline IZ1295 & $c k r-1(o k 2502)$ l; ckr-2(tm3082) III; ufls104 & \\
\hline IZ2544 & lin-15(n765ts); ufEx942 & 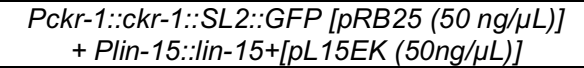 \\
\hline IZ1908 & ufls141 & $\begin{array}{c}\text { pCKR-1::CKR-1::SL2::mCherry } \\
{[p D T 205(40 \mathrm{ng} / \mu L)]}\end{array}$ \\
\hline IZ2065 & ufls148 & 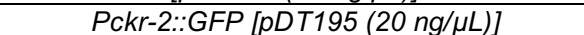 \\
\hline IZ2251 & ufls141; ufls148 & Pckr-1::ckr-1::SL2::mCherry; Pckr-2::GFP \\
\hline IZ2633 & ufEx942; ufls43 & $\begin{array}{c}\text { Pckr-1::ckr-1::SL2::GFP, Pacr-2::mCherry } \\
\text { [pPRB6 (30ng/ } \mu L)]\end{array}$ \\
\hline IZ2280 & ufls141; vs/s48 & Pckr-1::ckr-1::SL2::mCherry; Punc-17::GFP \\
\hline IZ2468 & ufls141; akEx263 & Pckr-1::ckr-1::SL2::mCherry; Punc-4::GFP \\
\hline IZ2245 & ufls141; oxls12 & Pckr-1::ckr-1::SL2::mCherry; Punc-47::GFP \\
\hline IZ2246 & ufls141; kyls51 & Pckr-1::ckr-1::SL2::mCherry; Podr-2(2b)::GFP \\
\hline IZ2454 & ufls141; ufEx863 & Pckr-1::ckr-1::SL2::mCherry; Podr-2(18)::GFP \\
\hline IZ2248 & ufls141; mgls18 & Pckr-1::ckr-1::SL2::mCherry; Pttx-3::GFP \\
\hline IZ2249 & ufls141; oyls18 & Pckr-1::ckr-1::SL2::mCherry; Pgcy-8::GFP \\
\hline IZ2250 & ufls141; oyls 14 & Pckr-1::ckr-1::SL2::mCherry; Psra-6::GFP \\
\hline IZ2447 & ufls141; otls337 & Pckr-1::ckr-1::SL2::mCherry; Plad-2::GFP \\
\hline IZ2499 & ufls141; zfls6 & Pckr-1::ckr-1::SL2::mCherry; Plgc-55::GFP \\
\hline IZ3533 & ufls141; ufEx1485 & 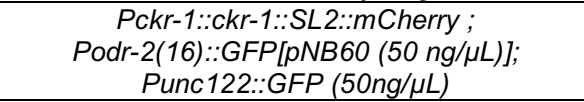 \\
\hline IZ3591 & ufis141; ufEx1504 & 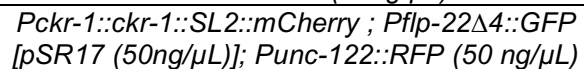 \\
\hline IZ2635 & ufEx942; $d b E x 721$ & Pckr-1::ckr-1::SL2::GFP, Pnpr-4::RFP \\
\hline IZ2455 & ufls141; adEx1616 & Pckr-1::ckr-1::SL2::mCherry; Pser-4::GFP \\
\hline IZ2459 & ufls $141 ;$ njls 10 & Pckr-1::ckr-1::SL2::mCherry; Pglr-3::GFP \\
\hline IZ2504 & ufls141; otls123 & Pckr-1::ckr-1::SL2::mCherry; Psra-11::GFP \\
\hline IZ2546 & ufls $141 ; \mathrm{mg} / \mathrm{s} 42$ & Pckr-1::ckr-1::SL2:::mCherry; Ptph-1::GFP \\
\hline IZ2277 & ufEx802 & $\begin{array}{l}\text { Pckr-1::genomic ckr-1 [pDT112 (100ng/ } / \mathrm{LL})] \\
\quad+\text { pHP6 [Plgc-11::GFP (50ng/ } \mu \mathrm{L})]\end{array}$ \\
\hline IZ2399 & nlp-12(ok335)l; ufEx802 & \\
\hline IZ3201 & ufEx953 & $\begin{array}{c}\text { Prgef-1::ckr-1 minigene [pRB20 }(25 n g / \mu L)] \\
+ \text { Plgc-11::GFP (50ng/ } \mu \mathrm{L})\end{array}$ \\
\hline IZ3217 & ufEx689 & 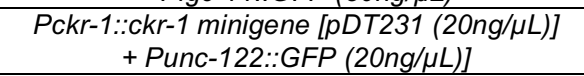 \\
\hline IZ3198 & ufEx962 & 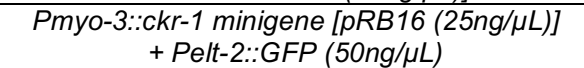 \\
\hline IZ3211 & ufEx1309 & $\begin{array}{l}\text { Punc-17 }:: \text { ckr-1 minigene }[p R B 14(50 n g / \mu L)] \\
+ \text { Plgc-11::GFP }[p H P 6(50 n g / \mu L)]\end{array}$ \\
\hline IZ3212 & ufEx1310 & $\begin{array}{l}\text { Punc-47::ckr-1 minigene }[p R B 15(25 n g / \mu L)] \\
\quad+\text { Plgc-11::GFP }[p H P 6(50 \mathrm{ng} / \mu L)]\end{array}$ \\
\hline IZ3197 & ufEx930 & $\begin{array}{c}\text { Plgc-55::ckr-1 minigene }[p R B 17(25 \mathrm{ng} / \mu \mathrm{L})] \\
+\mathrm{pHP6}(50 \mathrm{ng} / \mu \mathrm{L})]\end{array}$ \\
\hline IZ3203 & ufEx1003 & 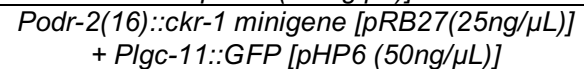 \\
\hline
\end{tabular}


bioRxiv preprint doi: https://doi.org/10.1101/2020.04.27.064550; this version posted June 28, 2021. The copyright holder for this preprint (which was not certified by peer review) is the author/funder. All rights reserved. No reuse allowed without permission.

\begin{tabular}{|c|c|c|}
\hline IZ3650 & ufEx1538 & $\begin{array}{c}\text { Pflp-22( } \Delta 4):: c k r-1 \text { minigene }[p S R 37(50 \mathrm{ng} / \mu \mathrm{L})] \\
+ \text { Plgc-11::GFP }[\mathrm{pHP} 6(50 \mathrm{ng} / \mu \mathrm{L})]\end{array}$ \\
\hline IZ3210 & ufEx1180 & $\begin{array}{l}\text { Pgcy-28d::ckr-1 minigene [pRB29 }(25 \mathrm{ng} / \mu \mathrm{L})] \\
+ \text { Plgc-11::GFP }[\mathrm{pHP} 6(50 \mathrm{ng} / \mu \mathrm{L})]\end{array}$ \\
\hline IZ3199 & ufEx1181 & $\begin{array}{c}\text { Podr-2(18)::ckr-1 minigene [pRB26 (25 } \\
n g / \mu L)]+ \text { Plgc-11::GFP [pHP6 (50 ng/ } / \mu L)]\end{array}$ \\
\hline IZ3231 & ufEx1196 & $\begin{array}{l}\text { Posm-6::ckr-1 minigene }[p N B 66(25 \mathrm{ng} / \mu \mathrm{L})] \\
\quad+\text { Plgc-11::m Cherry }[p B B 107(50 \mathrm{ng} / \mu \mathrm{L})]\end{array}$ \\
\hline IZ3200 & ufEx1197 & $\begin{array}{l}\text { Plim-4::ckr-1 minigene }[p N B 67(25 \mathrm{ng} / \mu \mathrm{L})] \\
+ \text { Plgc-11::m Cherry }[\mathrm{pBB} 107(50 \mathrm{ng} / \mu \mathrm{L})]\end{array}$ \\
\hline IZ3222 & ufEx1234 & $\begin{array}{l}\text { Pnpr-9::ckr-1 minigene }[p N B 61(25 \mathrm{ng} / \mu \mathrm{L})] \\
\quad+\text { Plgc-11::GFP }[\mathrm{pHP} 6(80 \mathrm{ng} / \mu \mathrm{L})]\end{array}$ \\
\hline IZ2461 & ckr-1(ok2502)l; ufEx911 & $\begin{array}{l}\text { Pckr-1::ckr-1 [pDT231 }(5 \mathrm{ng} / \mu \mathrm{L})] \\
+ \text { Plgc-11::GFP [pHP6 }(50 \mathrm{ng} / \mu \mathrm{L})]\end{array}$ \\
\hline IZ3112 & ckr-1(ok2502) l; ufEx1247 & $\begin{array}{l}\text { Podr-2(16)::ckr-1 minigene }(5 n g / \mu L) \\
+ \text { Plgc-11::GFP }[p H P 6(80 \mathrm{ng} / \mu \mathrm{L})]\end{array}$ \\
\hline IZ3116 & ckr-1(ok2502) l; ufEx1250 & 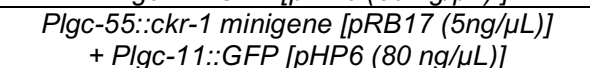 \\
\hline IZ3890 & $c k r-1(o k 2502)$ l; ufEx1646 & 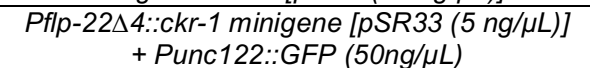 \\
\hline IZ3875 & ufEx1638 & 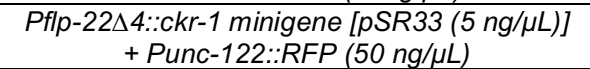 \\
\hline IZ3587 & ufEx1518 & 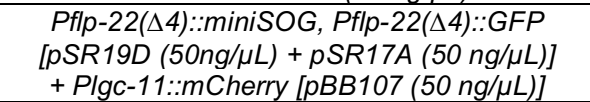 \\
\hline IZ3701 & ufEx802; ufEx1518 & $\begin{array}{c}\text { Pckr-1::ckr-1 genomic; Pflp-22( } \Delta 4):: \text { miniSOG; } \\
\text { Pflp-22 } 4:: G F P\end{array}$ \\
\hline IZ1782 & lite-1(ce314) X; ufls140 & $\begin{array}{l}\text { Pnlp-12::ChR::GFP [pCL28 (50 ng/ } / \mu L)] \\
\quad+\text { Plgc-11::GFP }[p H P 6(30 \mathrm{ng} / \mu \mathrm{L})]\end{array}$ \\
\hline IZ1779 & nlp-12(ok335) l; lite-1(ce314) X; ufls140 & \\
\hline IZ1968 & $\begin{array}{l}c k r-1(o k 2502) \text { l; ckr-2(tm3082) III; } \\
\text { lite-1(ce314) X; ufls140 }\end{array}$ & \\
\hline IZ1777 & ckr-1(ok2502) l; lite-1(ce314) X; ufls140 & \\
\hline IZ1781 & ckr-2(tm3082) III; lite-1(ce314) X; ufls140 & \\
\hline IZ3645 & ufls186 & 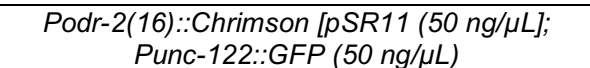 \\
\hline IZ3598 & ufEx1522 & 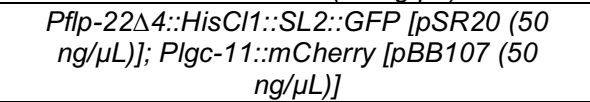 \\
\hline IZ3788 & ufEx1584 & 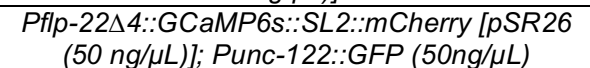 \\
\hline IZ4208 & lin-15(n765ts)X; ufEx1784 & 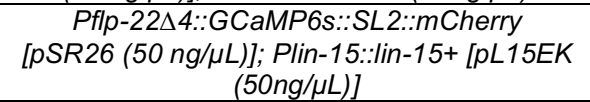 \\
\hline IZ3824 & ufEx1584; ckr-1(ok2502) I & \\
\hline IZ4164 & ufEx1759; ckr-1(ok2502) I & $\begin{array}{l}\text { Pckr-2::ckr-1 minigene }[p S R 81(5 \mathrm{ng} / \mu \mathrm{L})] ; \\
\text { Pinx-6::GFP [pDO125 (50 ng/mL)] }\end{array}$ \\
\hline IZ4255 & ufEx1815; nlp-12(If) I & $\begin{array}{l}\text { Pser-2(prom3)::nlp-12 [pCL131 (5 ng/ } \mu \mathrm{L})] \text {; } \\
\text { Plgc-11::mCherry[pBB107 (50ng/ } \mu \mathrm{L})]\end{array}$ \\
\hline
\end{tabular}




\section{Table S2}

Identification (method of ID, marker and strain indicated for each neuron) to determine ckr-1 expressing neurons. * Indicated strains were crossed into ufls141 (Pckr-1::ckr-1::SL2::GFP) to generate strains to determine colocalization. ${ }^{\#}+$ or - indicates presence or absence of $c k r-1$ expression in identified neuron.

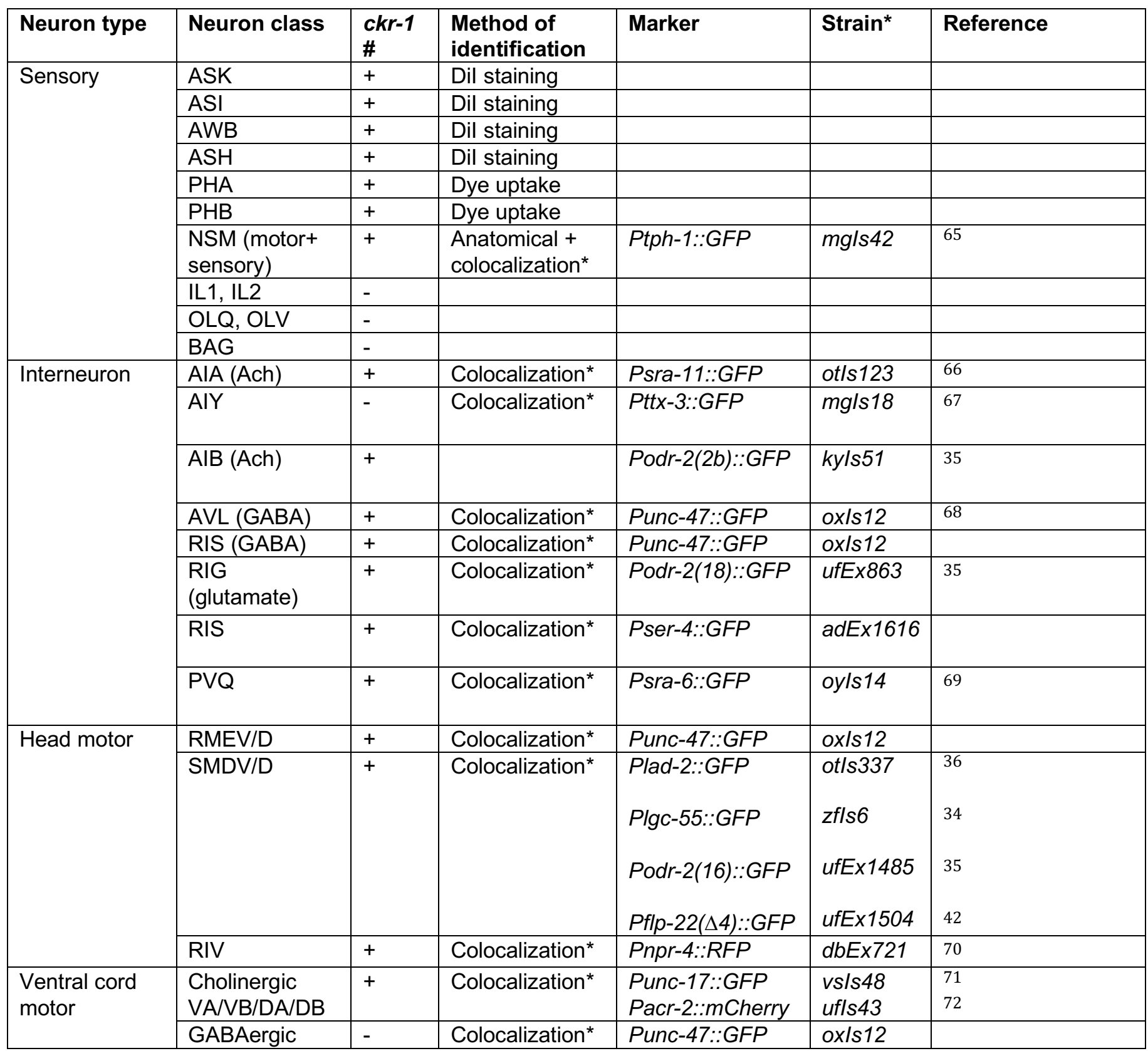

${ }^{*}$ Indicated strains were crossed into ufls141 to generate strains to determine colocalization \# + indicates ckr-1 expression, - indicates absence 


\section{Table S3}

\begin{tabular}{|c|c|c|}
\hline Promoter & Expression & References \\
\hline Pckr-1 & ckr-1 native promoter (3564 bp) & \\
\hline Prgef-1 & Pan-neuronal & 73 \\
\hline Punc-17 $\beta$ & Restricted expression in cholinergic motor neurons (VNC) & 68 \\
\hline Punc-47 & All GABAergic neurons & 35 \\
\hline Pmyo-3 & Muscles & 34 \\
\hline Podr-2(16) & SMD, RME & 42 \\
\hline Plgc-55 & SMD, IL1, AVB, RMD, neck muscles & 74 \\
\hline Pflp-22 4 SMD, URX & 34 \\
\hline Pgcy-28.d & AIA & \\
\hline $\begin{array}{c}\text { Plim-4(s) (lim-4 } \\
\text { promoter fragment } \\
\text { from -3328 to -2174 } \\
\text { upstream of start) }\end{array}$ & RIV & 75 \\
\hline Pnpr-9 & AIB & 35 \\
\hline Podr-2(18) & RIG, SMB & 76 \\
\hline Posm-6 & ADE, amphid neurons, AQR, CEM, CEP, HOA, HOB, IL1, IL2, OLL, \\
\hline
\end{tabular}

Promoters used in ckr-1(OE) screen (Fig. 5C) indicating expression pattern. ${ }^{* \star}$ Bold indicates neurons where $c k r-1$ is expressed. 


\section{Table S4}

Plasmid constructs used in cell specific $c k r-1(O E)$ screen or cell-specifc rescue (Fig. 5C, 7A). For cell specific overexpression or rescue of $c k r-1, c k r-1$ minigene was expressed under indicated promoters. Entry vectors containing promoters recombined with destination vectors pRB12 or pRB13 for cell-specific overexpression or rescue of $c k r-1$.

\begin{tabular}{|c|c|}
\hline Plasmid & $c k r-1$ expression construct \\
\hline pRB31 & Pacr-2::ckr-1 \\
\hline pRB14 & Punc-17ß::ckr-1 \\
\hline pRB16 & Pmyo-3::ckr-1 \\
\hline pRB30 & Plad-2::ckr-1 \\
\hline pRB17 & Plgc-55::ckr-1 \\
\hline pRB27 & Podr-2(16)::ckr-1 \\
\hline pSR33 & Pflp-22\4::ckr-1 \\
\hline pRB18 & Pglr-2::ckr-1 \\
\hline pRB20 & PF25B3.3::ckr-1 \\
\hline pRB21 & Pttr-39::ckr-1 \\
\hline pRB22 & Pdel-1::ckr-1 \\
\hline pRB23 & Punc-129::ckr-1 \\
\hline pRB24 & Plim-6 Intron 4::ckr-1 \\
\hline pRB26 & Podr-2(18)::ckr-1 \\
\hline pRB28 & Ptph-1::ckr-1 \\
\hline pRB29 & Pgcy-28d::ckr-1 \\
\hline pNB66 & Posm-6::ckr-1 \\
\hline pNB67 & Plim-4::ckr-1 \\
\hline pNB61 & Pnpr-9::ckr-1 \\
\hline
\end{tabular}


bioRxiv preprint doi: https://doi.org/10.1101/2020.04.27.064550; this version posted June 28, 2021. The copyright holder for this preprint (which was not certified by peer review) is the author/funder. All rights reserved. No reuse allowed without permission.

\section{Table S5}

Promoter lengths and primer information for promoters used

\begin{tabular}{|c|}
\hline $\begin{array}{l}\text { Pckr-1 } \\
\text { 3564bp promoter region of } c k r-1 \text { amplified from genomic DNA } \\
\text { OMF2159 (Forward primer):-CTGCAGGATGGAGATTCAATCAGC } \\
\text { OMF2160 (Reverse primer): TGTGTATCTGAAAATTTTTAATTTTAAA }\end{array}$ \\
\hline $\begin{array}{l}\text { Pckr-2 } \\
8.6 \mathrm{~kb} \text { promoter region of } c k r-2 \text { amplified from genomic DNA } \\
\text { OMF1067 (Forward primer): GCATGCGGGTGTGATAAGTGCAATGAAGTGG } \\
\text { OMF1068 (Reverse primer): ACGTACCGGTTCCTCCTGATGTACCGTTGACATTGTGG }\end{array}$ \\
\hline $\begin{array}{l}\text { Plgc-55 } \\
\text { 2663bp amplified from plasmid lgc55_2663-0_pPD95_75 (Mark Alkema) } \\
\text { OMF1019 (Forward primer): ATGTCTGCCCCATCACCAGTG } \\
\text { OMF1020 (Reverse primer): TTCATTTCGACATCTATTTGCCAATA }\end{array}$ \\
\hline $\begin{array}{l}\text { Podr-2(16) } \\
\text { 3.2 kb promoter fragment amplified from genomic DNA } \\
\text { OMF1878 (Forward primer): ATGGGAATGGGGCAAAT } \\
\text { OMF1879 (Reverse primer): CGGGCATCCCGACAAACTGT }\end{array}$ \\
\hline $\begin{array}{l}\text { Plad-2 } \\
4.5 \mathrm{~kb} \text { upstream of lad-2 ATG amplified from genomic DNA } \\
\text { OMF1876 (Forward primer): ATTTTTTCTGTGTTCCACTAA } \\
\text { OMF1920 (Reverse primer): TGTTGGAAAAATCCAAAAAAAAAGTCTGC }\end{array}$ \\
\hline$P f l p-22(\Delta 4)$ \\
\hline $\begin{array}{l}\text { 1532bp promoter fragment was amplified from genomic DNA based on Yeon et. al } 2018 .{ }^{37} \\
\text { OMF2610 (Forward primer): TGCAGGAAACACCACCTAGTATATAAT } \\
\text { OMF2611 (Reverse primer): TGCAAGCTTAGAGTACAACGGCGA }\end{array}$ \\
\hline $\begin{array}{l}\text { Pmyo-3 } \\
\text { Amplified myo-3 promoter(2344bp) from pBB38 (Pmyo-3::acr-2 cDNA) } \\
\text { OMF763 (Forward primer): CGGCTATAATAAGTCTTGAATAA } \\
\text { OMF764 (Reverse primer): CCTGAAAATTAGACGGTAAAAGTG }\end{array}$ \\
\hline $\begin{array}{l}\text { Punc17 } \beta \\
\text { unc-17 } \beta \text { promoter (485bp) amplified from plasmid pRM\#621 } \\
\text { OMF1594 (Forward primer): TTGGTTTTCACAATTTCTGGTTTT } \\
\text { OMF1595 (Reverse primer): TTGAACAAGAGATGCGGAAAATAGAAAGA }\end{array}$ \\
\hline $\begin{array}{l}\text { Punc-47 } \\
\text { unc-47 promoter (1158bp) amplified from genomic DNA } \\
\text { OMF658 (Forward primer): AGTCGAAAGTCGGTGGCAAG } \\
\text { OMF659 (Reverse primer): GTCGTCTCACAGGAAAGACAGAT }\end{array}$ \\
\hline $\begin{array}{l}\text { Podr-2(18) } \\
\text { 2.4 kb promoter fragment amplified from genomic DNA } \\
\text { OMF1015 (Forward primer): GAACAGGGTCTCTCACAGTTTGTCG } \\
\text { OMF1016 (Reverse primer):_CCATCAGCCAAATGTAGGCTCGG }\end{array}$ \\
\hline $\begin{array}{l}\text { Pnpr-9 } \\
\text { npr-9 promoter (2305bp) amplified from genomic DNA } \\
\text { OMF1353 (Forward primer): CAATGCAGAAGAAGACTCTTCATCC } \\
\text { OMF1544 (Reverse primer): GACATTTCCCAACGACATTTCCC }\end{array}$ \\
\hline $\begin{array}{l}\text { Pgcy-28d } \\
2841 \text { bp promoter for gcy-28d amplified from genomic DNA. } \\
\text { OMF1884 (Forward primer): TACAATTGTAGTGAGCTTCG } \\
\text { OMF1885 (Reverse primer): TTCGCACTCATCTCACCATTCC }\end{array}$ \\
\hline $\begin{array}{l}\text { Plim-4 } \\
\text { lim-4 promoter fragment from }-3328 \text { to }-2174 \text { upstream of start was amplified from plasmid } \\
\text { [Plim-4(-3328 to -2174)::NLSwCherry::SL2::GCaMP6s] (Mark Alkema) } \\
\text { OMF2166 (Forward primer): AAGCTTTGATTAGAAATTGAGTTC } \\
\text { OMF2167 (Reverse primer): ACAAGCCGCTCAGTTTTGATCTAAAAT }\end{array}$ \\
\hline $\begin{array}{l}\text { Posm-6 } \\
2083 \text { bp promoter region from Posm-6::GFP (Claire Benard) } \\
\text { OMF2227 (Forward primer): CTTGCATGTTATGGATACTCTGAAT } \\
\text { OMF2228 (Reverse primer): TTTTCGTCTGAAAATGGAGGCATAG }\end{array}$ \\
\hline $\begin{array}{l}\text { Ptph-1 } \\
\text { tph-1 promoter was amplified from Ptph-1::unc-2 (Mark Alkema) } \\
\text { OMF926 (Forward primer): GGTGGTCTTCCCGCTTGCAATAC } \\
\text { OMF927 (Reverse primer): ATGATTGAAGAGAGCAATGCTAC }\end{array}$ \\
\hline $\begin{array}{l}\text { Pser-2prom } 3 \\
4101 \text { bp promoter region of the ser-2 promoter (ser-2prom } 3)^{33} \text { was amplified from genomic DNA } \\
\text { OMF3149 Forward primer: GTAAAGTTAGTAAATAACTGCTA } \\
\text { OMF3150 Reverse primer: TATGTGTTGTGATGTCACAAAATATG }\end{array}$ \\
\hline
\end{tabular}




\section{Supplementary Videos}

Video S1. Representative 20 second video showing locomotion on food of animal overexpressing $n / p-12$. Video has been sped up $4 \mathrm{X}$.

Video S2. Representative 20 second video showing locomotion of wild type animal during area restricted search (0-5 minutes off food). Video has been sped up 4X.

Video S3. Representative 20 second video showing locomotion of wild type animal during dispersal (30-35 minutes off food). Video has been sped up 4X.

Video S4. Representative 20 second video showing locomotion on food of animal overexpressing $c k r-1$. Video has been sped up 4X.

Video S5. Representative 20 second video showing locomotion on food of animal overexpressing $c k r-1$ in the SMD motor neurons. Video has been sped up 4X.

Video S6. Representative 20 second video showing locomotion on food of animal in the absence (left) and during SMD photostimulation (right). Video has been sped up 4X.

Video S7. Representative 20 second video showing single worm tracking of wild type animal during basal locomotion on food to analyze body bending and head bending. Video has been sped up $4 X$.

Video S8. Representative 20 second video showing tracking locomotion of animal overexpressing $n / p-12$ in Wormlab to analyze body bending. Video has been sped up 4X. Video S9. Representative 20 second video showing simultaneous post-hoc tracking of mCherry and GCaMP6s fluorescence for ratiometric calcium imaging analysis. Video has been sped up $4 X$. 


\section{References}

1. Bailer, U. \& Kaye, W. A Review of Neuropeptide and Neuroendocrine Dysregulation in Anorexia and Bulimia Nervosa. Current Drug Targets -CNS and Neurological Disorders 2, 53-59 (2003).

2. Pomrenze, M. B. et al. Dissecting the Roles of GABA and Neuropeptides from Rat Central Amygdala CRF Neurons in Anxiety and Fear Learning. Cell Reports 29, 13-21.e4 (2019).

3. Kormos, V. \& Gaszner, B. Role of neuropeptides in anxiety, stress, and depression: From animals to humans. Neuropeptides 47, 401-419 (2013).

4. Zhang, L., Hernandez-Sanchez, D. \& Herzog, H. Regulation of Feeding-Related Behaviors by Arcuate Neuropeptide Y Neurons. Endocrinology 160, 1411-1420 (2019).

5. West, K. S. \& Roseberry, A. G. Neuropeptide-Y alters VTA dopamine neuron activity through both pre- and postsynaptic mechanisms. J Neurophysiol 118, 625-633 (2017).

6. Bhattacharya, R. et al. A Conserved Dopamine-Cholecystokinin Signaling Pathway Shapes ContextDependent Caenorhabditis elegans Behavior. Plos Genet 10, e1004584 (2014).

7. Hums, I. et al. Regulation of two motor patterns enables the gradual adjustment of locomotion strategy in Caenorhabditis elegans. Elife 5, e14116 (2016).

8. Oranth, A. et al. Food Sensation Modulates Locomotion by Dopamine and Neuropeptide Signaling in a Distributed Neuronal Network. Neuron 100, 1414-1428.e10 (2018).

9. Janssen, T. et al. Discovery of a Cholecystokinin-Gastrin-Like Signaling System in Nematodes. Endocrinology 149, 2826-2839 (2008).

10. Janssen, T., Meelkop, E., Nachman, R. J. \& Schoofs, L. Evolutionary Conservation of the Cholecystokinin/Gastrin Signaling System in Nematodes. Ann Ny Acad Sci 1163, 428-432 (2009).

11. Peeters, L. et al. A pharmacological study of NLP-12 neuropeptide signaling in free-living and parasitic nematodes. Peptides 34, 82-87 (2012).

12. Lee, S. Y. \& Soltesz, I. Cholecystokinin: A multi-functional molecular switch of neuronal circuits. Dev Neurobiol 71, 83-91 (2011).

13. Nishimura, S. et al. Functional Synergy between Cholecystokinin Receptors CCKAR and CCKBR in Mammalian Brain Development. Plos One 10, e0124295 (2015).

14. Ballaz, S. The unappreciated roles of the cholecystokinin receptor $\operatorname{CCK}(1)$ in brain functioning. Rev Neuroscience 28, 573-585 (2017).

15. Saito, A., Sankaran, H., Goldfine, I. \& Williams, J. Cholecystokinin receptors in the brain: characterization and distribution. Science 208, 1155-1156 (1980).

16. Frooninckx, L. et al. Neuropeptide GPCRs in C. elegans. Front Endocrinol 3, 167 (2012). 
17. Paiva, V. H., Geraldes, P., Ramírez, I., Garthe, S. \& Ramos, J. A. How area restricted search of a pelagic seabird changes while performing a dual foraging strategy. Oikos 119, 1423-1434 (2010).

18. Sommerfeld, J., Kato, A., Ropert-Coudert, Y., Garthe, S. \& Hindell, M. A. Foraging Parameters Influencing the Detection and Interpretation of Area-Restricted Search Behaviour in Marine Predators: A Case Study with the Masked Booby. Plos One 8, e63742 (2013).

19. Hills, T. T., Kalff, C. \& Wiener, J. M. Adaptive Lévy Processes and Area-Restricted Search in Human Foraging. Plos One 8, e60488 (2013).

20. Weimerskirch, H., Pinaud, D., Pawlowski, F. \& Bost, C. Does Prey Capture Induce Area-Restricted Search? A Fine-Scale Study Using GPS in a Marine Predator, the Wandering Albatross. Am Nat 170, 734-743 (2007).

21. Bell, W. Searching Behavior Patterns In Insects. Annu Rev Entomol 35, 447-467 (1990).

22. Bailey, H. et al. Empirical evidence that large marine predator foraging behavior is consistent with area-restricted search theory. Ecology 100, (2019).

23. Marques, J. C., Li, M., Schaak, D., Robson, D. N. \& Li, J. M. Internal state dynamics shape brainwide activity and foraging behaviour. Nature 577, 239-243 (2020).

24. Gray, J. M., Hill, J. J. \& Bargmann, C. I. A circuit for navigation in Caenorhabditis elegans. Proc National Acad Sci 102, 3184-3191 (2005).

25. Hills, T., Brockie, P. J. \& Maricq, A. V. Dopamine and Glutamate Control Area-Restricted Search Behavior in Caenorhabditis elegans. J Neurosci 24, 1217-1225 (2004).

26. Wakabayashi, T., Kitagawa, I. \& Shingai, R. Neurons regulating the duration of forward locomotion in Caenorhabditis elegans. Neurosci Res 50, 103-111 (2004).

27. Calhoun, A. J., Chalasani, S. H. \& Sharpee, T. O. Maximally informative foraging by Caenorhabditis elegans. Elife 3, e04220 (2014).

28. White, J. G., Southgate, E., Thomson, J. N. \& Brenner, S. The structure of the ventral nerve cord of Caenorhabditis elegans. Philosophical Transactions Royal Soc Lond Ser B Biological Sci 275, 327-48 (1976).

29. Hu, Z., Pym, E. C. G., Babu, K., Vashlishan Murray, A. B. \& Kaplan, J. M. A NeuropeptideMediated Stretch Response Links Muscle Contraction to Changes in Neurotransmitter Release. Neuron 71, 92-102 (2011).

30. Hu, Z., Vashlishan-Murray, A. B. \& Kaplan, J. M. NLP-12 Engages Different UNC-13 Proteins to Potentiate Tonic and Evoked Release. J Neurosci 35, 1038-1042 (2015).

31. Caers, J. et al. Characterization of G Protein-coupled Receptors by a Fluorescence-based Calcium Mobilization Assay. J Vis Exp Jove 51516 (2014) doi:10.3791/51516. 
32. Nagel, G. et al. Channelrhodopsin-2, a directly light-gated cation-selective membrane channel. Proc National Acad Sci 100, 13940-13945 (2003).

33. Tao, L. et al. Parallel Processing of Two Mechanosensory Modalities by a Single Neuron in C. elegans. Dev Cell (2019) doi:10.1016/j.devcel.2019.10.008.

34. Pirri, J. K., McPherson, A. D., Donnelly, J. L., Francis, M. M. \& Alkema, M. J. A Tyramine-Gated Chloride Channel Coordinates Distinct Motor Programs of a Caenorhabditis elegans Escape Response. Neuron 62, 526-538 (2009).

35. Chou, J. H., Bargmann, C. I. \& Sengupta, P. The Caenorhabditis elegans odr-2 gene encodes a novel Ly-6-related protein required for olfaction. Genetics 157, 211-24 (2001).

36. Wang, X. et al. The C. elegans L1CAM homologue LAD-2 functions as a coreceptor in MAB20/Sema2 mediated axon guidance. J Cell Biology 180, 233-46 (2008).

37. Kaletsky, R. et al. Transcriptome analysis of adult Caenorhabditis elegans cells reveals tissue-specific gene and isoform expression. Plos Genet 14, e1007559 (2018).

38. Hendricks, M., Ha, H., Maffey, N. \& Zhang, Y. Compartmentalized calcium dynamics in a C. elegans interneuron encode head movement. Nature 487, 99-103 (2012).

39. Shen, Y. et al. An extrasynaptic GABAergic signal modulates a pattern of forward movement in Caenorhabditis elegans. Elife 5, e14197 (2016).

40. Kocabas, A., Shen, C.-H., Guo, Z. V. \& Ramanathan, S. Controlling interneuron activity in Caenorhabditis elegans to evoke chemotactic behaviour. Nature 490, 273-277 (2012).

41. Kaplan, H. S., Thula, O. S., Khoss, N. \& Zimmer, M. Nested Neuronal Dynamics Orchestrate a Behavioral Hierarchy across Timescales. Neuron (2019) doi:10.1016/j.neuron.2019.10.037.

42. Yeon, J. et al. A sensory-motor neuron type mediates proprioceptive coordination of steering in C. elegans via two TRPC channels. Plos Biol 16, e2004929 (2018).

43. Xu, S. \& Chisholm, A. D. Highly efficient optogenetic cell ablation in C. elegans using membranetargeted miniSOG. Sci Rep-uk 6, 21271 (2016).

44. Klapoetke, N. C. et al. Independent optical excitation of distinct neural populations. Nat Methods 11, 338-346 (2014).

45. Miyasaka, K. \& Funakoshi, A. Cholecystokinin and cholecystokinin receptors. J Gastroenterol 38, 1 13 (2003).

46. Li, X. et al. Cholecystokinin from the entorhinal cortex enables neural plasticity in the auditory cortex. Cell Res 24, 307-330 (2014).

47. Chen, X. et al. Cholecystokinin release triggered by NMDA receptors produces LTP and sound-sound associative memory. P Natl Acad Sci Usa 116, 6397-6406 (2019). 
48. Crosby, K. M. et al. Cholecystokinin Switches the Plasticity of GABA Synapses in the Dorsomedial Hypothalamus via Astrocytic ATP Release. J Neurosci 38, 8515-8525 (2018).

49. Broekmans, O. D., Rodgers, J. B., Ryu, W. S. \& Stephens, G. J. Resolving coiled shapes reveals new reorientation behaviors in C. elegans. Elife 5, e17227 (2016).

50. White, J. Clues to basis of exploratory behaviour of the C. elegans snout from head somatotropy. Philosophical Transactions Royal Soc Lond Ser B Biological Sci 373, 20170367 (2018).

51. Ouellette, M.-H., Desrochers, M. J., Gheta, I., Ramos, R. \& Hendricks, M. A Gate-and-Switch Model for Head Orientation Behaviors in Caenorhabditis elegans. Eneuro 5, ENEURO.0121-18.2018 (2018).

52. Pierce-Shimomura, J. T., Morse, T. M. \& Lockery, S. R. The Fundamental Role of Pirouettes in Caenorhabditis elegans Chemotaxis. J Neurosci 19, 9557-9569 (1999).

53. Gao, S. et al. Excitatory motor neurons are local oscillators for backward locomotion. Elife 7, e29915 (2018).

54. Xu, T. et al. Descending pathway facilitates undulatory wave propagation in Caenorhabditis elegans through gap junctions. Proc National Acad Sci 115, 201717022 (2018).

55. Sawin, E. R., Ranganathan, R. \& Horvitz, H. R. C. elegans Locomotory Rate Is Modulated by the Environment through a Dopaminergic Pathway and by Experience through a Serotonergic Pathway. Neuron 26, 619-631 (2000).

56. Rehfeld, J. F. Cholecystokinin-From Local Gut Hormone to Ubiquitous Messenger. Front Endocrinol 8, 47 (2017).

57. Liddle, R. A. CHOLECYSTOKININ CELLS. Annu Rev Physiol 59, 221-242 (1997).

58. Chandra, R. \& Liddle, R. A. Cholecystokinin. Curr Opin Endocrinol Diabetes Obes 14, 63-67 (2007).

59. Noble, F. \& Roques, B. P. Handbook of Neurochemistry and Molecular Neurobiology, Neuroactive Proteins and Peptides. 545-571 (2006) doi:10.1007/978-0-387-30381-9_24.

60. Yemini, E., Kerr, R. A. \& Schafer, W. R. Preparation of Samples for Single-Worm Tracking. Cold Spring Harb Protoc 2011, pdb.prot066993 (2011).

61. Yemini, E., Jucikas, T., Grundy, L. J., Brown, A. E. X. \& Schafer, W. R. A database of Caenorhabditis elegans behavioral phenotypes. Nat Methods 10, 877-879 (2013).

62. Peymen, K. et al. Myoinhibitory peptide signaling modulates aversive gustatory learning in Caenorhabditis elegans. Plos Genet 15, e1007945 (2019).

63. Sinay, E. V. et al. Evolutionarily conserved TRH neuropeptide pathway regulates growth in Caenorhabditis elegans. Proc National Acad Sci 114, E4065-E4074 (2017). 
64. Dereeper, A. et al. Phylogeny.fr: robust phylogenetic analysis for the non-specialist. Nucleic Acids Res 36, W465-W469 (2008).

65. Sze, J. Y., Victor, M., Loer, C., Shi, Y. \& Ruvkun, G. Food and metabolic signalling defects in a Caenorhabditis elegans serotonin-synthesis mutant. Nature 403, 560-564 (2000).

66. Altun-Gultekin, Z. et al. A regulatory cascade of three homeobox genes, ceh-10, ttx-3 and ceh-23, controls cell fate specification of a defined interneuron class in C. elegans. Dev Camb Engl 128, 1951-69 (2001).

67. Hobert, O. et al. Regulation of Interneuron Function in the C. elegans Thermoregulatory Pathway by the ttx-3 LIM Homeobox Gene. Neuron 19, 345-357 (1997).

68. McIntire, S. L., Reimer, R. J., Schuske, K., Edwards, R. H. \& Jorgensen, E. M. Identification and characterization of the vesicular GABA transporter. Nature 389, 870-876 (1997).

69. Aurelio, O., Boulin, T. \& Hobert, O. Identification of spatial and temporal cues that regulate postembryonic expression of axon maintenance factors in the $\mathrm{C}$. elegans ventral nerve cord. Development 130, 599-610 (2003).

70. Cohen, M. et al. Coordinated Regulation of Foraging and Metabolism in C. elegans by RFamide Neuropeptide Signaling. Cell Metab 9, 375-385 (2009).

71. Alfonso, A., Grundahl, K., Duerr, J., Han, H. \& Rand, J. The Caenorhabditis elegans unc-17 gene: a putative vesicular acetylcholine transporter. Science 261, 617-619 (1993).

72. Petrash, H. A., Philbrook, A., Haburcak, M., Barbagallo, B. \& Francis, M. M. ACR-12 Ionotropic Acetylcholine Receptor Complexes Regulate Inhibitory Motor Neuron Activity in Caenorhabditis elegans. J Neurosci 33, 5524-5532 (2013).

73. Charlie, N. K., Schade, M. A., Thomure, A. M. \& Miller, K. G. Presynaptic UNC-31 (CAPS) Is Required to Activate the Gas Pathway of the Caenorhabditis elegans Synaptic Signaling Network. Genetics 172, 943-961 (2006).

74. Shinkai, Y. et al. Behavioral Choice between Conflicting Alternatives Is Regulated by a Receptor Guanylyl Cyclase, GCY-28, and a Receptor Tyrosine Kinase, SCD-2, in AIA Interneurons of Caenorhabditis elegans. J Neurosci 31, 3007-3015 (2011).

75. Campbell, J. C., Polan-Couillard, L. F., Chin-Sang, I. D. \& Bendena, W. G. NPR-9, a Galanin-Like G-Protein Coupled Receptor, and GLR-1 Regulate Interneuronal Circuitry Underlying Multisensory Integration of Environmental Cues in Caenorhabditis elegans. Plos Genet 12, e1006050 (2016).

76. Collet, J., Spike, C. A., Lundquist, E. A., Shaw, J. E. \& Herman, R. K. Analysis of osm-6, a gene that affects sensory cilium structure and sensory neuron function in Caenorhabditis elegans. Genetics $\mathbf{1 4 8}$, 187-200 (1998). 